\title{
Some results on best proximity points of cyclic alpha-psi contractions in Menger probabilistic metric spaces
}

\author{
M. De la Sen ${ }^{1} \cdot$ Antonio F. Roldán $^{2}$
}

Received: 20 June 2016/ Accepted: 20 January 2017/Published online: 10 February 2017

(C) The Author(s) 2017. This article is published with open access at Springerlink.com

\begin{abstract}
This paper investigates properties of convergence of distances of $p$-cyclic $\alpha$ - $\psi$-type contractions on the union of the $p$ subsets of a space $X$ defining probabilistic metric spaces and Menger spaces. The paper also investigates the characterization of both Cauchy and $G$-Cauchy sequences which are convergent, in particular, to best proximity points. On the other hand, the existence and uniqueness of fixed points and best proximity points of $p$ cyclic $\alpha-\psi$-type contractions are also investigated. The fixed points of the $p$-composite self-mappings, which are obtained from the $p$-cyclic self-mapping restricted to each of the $p$ subsets in the cyclic disposal, are also investigated while a generalization and some illustrative examples are also given.
\end{abstract}

Keywords $p$-cyclic $\alpha-\psi$ contractions - Best proximity points - Probabilistic metric spaces - Menger spaces . Triangular norms

M. De la Sen

manuel.delasen@ehu.es

Antonio F. Roldán

afroldan@ujaen.es

1 Faculty of Science and Technology, Institute of Research and Development of Processes IIDP, University of the Basque Country, PO Box 644 de Bilbao. Barrio Sarriena, 48940 Leioa, Bizkaia, Spain

2 Department of Mathematics, University of Jaén, Paraje de las Lagunillas s/n, 23071 Jaén, Spain

\section{Introduction}

Fixed point theory in the framework of probabilistic metric spaces [1-4] is receiving important research attention. See, for instance, [2-4, 7-13]. In addition, Menger probabilistic metric spaces are a special case of the wide class of probabilistic metric spaces which are endowed with a triangular norm $[2,3,7,9,11,15,16,30]$. In probabilistic metric spaces, the deterministic notion of distance is considered to be probabilistic in the sense that, given any two points $x$ and $y$ of a metric space, a measure of the distance between them is a probabilistic metric $F_{x, y}(t)$, rather than the deterministic distance $d(x, y)$, which is interpreted as the probability of the distance between $x$ and $y$ being less than $t(t>0)$ [3].

Fixed point theorems in complete Menger spaces for probabilistic concepts of $B$ and $C$-contractions can be found in [2] together with a new notion of contraction, referred to as $(\Psi, C)$-contraction. Such a contraction was proved to be useful for multivalued mappings while it generalizes the previous concept of $C$-contraction. On the other hand, 2-cyclic $\varphi$-contractions on intersecting subsets of complete Menger spaces were discussed in [7] for contractions based on control $\varphi$-functions. See also [8]. It was found that fixed points are unique. In addition, $\varphi$-contractions in complete probabilistic Menger spaces have been also studied in [11] through the use of altering distances. See also $[14,26]$. On the other hand, probabilistic Banach spaces versus Fixed Point Theory were discussed in [10]. The concept of probabilistic complete metric space was adapted to the formalism of Banach spaces defined with norms being defined by triangular functions and under a suitable ordering in the considered space. In parallel, mixed monotone operators in such Banach spaces were discussed while the existence of coupled minimal and maximal fixed points for these operators was analyzed and discussed in 
detail. Further extensions to contractive mappings in complete fuzzy metric spaces using generalized distance distribution functions have been studied in [8,9] and references therein. The concept of altering distances was exploited in a very general context to derive fixed point results in [14], and extended later on in [15] to Menger probabilistic metric spaces. On the other hand, general fixed point theorems have been very recently obtained in [16] for two new classes of contractive mappings in Menger probabilistic metric spaces. The results have been established for $\alpha-\psi$ contractive mappings and for a generalized $\beta$-type one. It has also to be pointed out that the parallel background literature related to results on best proximity points and fixed points in cyclic mappings in metric and Banach spaces as well as topics related to common fixed points is exhaustive including studies of fixed point theory and applications in the fuzzy framework. See, for instance, [5, 6, 13, 17-27, 31-37] as well as references therein.

This paper investigates properties of convergence of distances of $p$-cyclic contractions on the union of the $p$ subsets of the abstract set $X$ defining the probabilistic metric spaces and the Menger spaces as well as the characterization of Cauchy and $G$-Cauchy sequences which converge to best proximity points of $p$-cyclic $\alpha-\psi$-type contractions. The existence and uniqueness of fixed points and best proximity points of $p$-cyclic $\alpha-\psi$-type contractions. The fixed points of the $p$-composite self-mappings, which are obtained from the cyclic self-mapping restricted to each of the $p$ subsets in the cyclic disposal, are also investigated while illustrative examples and a further generalization are also given.

Denote $\quad \mathbf{R}_{+}=\{z \in \mathbf{R}: z>0\}, \quad \mathbf{R}_{0+}=\mathbf{R}_{+} \cup\{0\}$, $\mathbf{Z}_{+}=\{z \in \mathbf{Z}: z>0\}, \quad \mathbf{Z}_{0+}=\mathbf{Z}_{+} \cup\{0\}$, $\bar{n}=\{1,2, \ldots, n\}$, and denote also by $\mathbf{L}$, the set of distance distribution functions $H: \mathbf{R} \rightarrow[0,1]$, [1], which are nondecreasing and left continuous such that $H(0)=0$ and $\sup _{t \in \mathbf{R}} H(t)=1$. Let $X$ be a nonempty set and let the probabilistic metric (or distance) $F: X \times X \rightarrow L$ a symmetric mapping from $X \times X$, where $X$ is an abstract set, to the set of distance distribution functions $L$ of the form $H: \mathbf{R} \rightarrow$ $[0,1]$ which are functions of elements $F_{x, y}$ for every $(x, y) \in X \times X$. Then, the ordered pair $(X, F)$ is a probabilistic metric space (PM) [2, 3, 29] if

1. $\forall x, y \in X\left(\left(F_{x, y}(t)=1 ; \forall t \in \mathbf{R}_{+}\right) \Leftrightarrow(x=y)\right)$
2. $F_{x, y}(t)=F_{y, x}(t) ; \forall x, y \in X, \forall t \in \mathbf{R}$

3. $\forall x, y, z \in X ; \quad \forall t_{1}, t_{2} \in \mathbf{R}_{+}\left(\left(F_{x, y}\left(t_{1}\right)\right.\right.$

$$
\left.\left.=F_{y, z}\left(t_{2}\right)=1\right) \Rightarrow\left(F_{x, z}\left(t_{1}+t_{2}\right)=1\right)\right)
$$

A particular distance distribution function $F_{x, y} \in L$ is a probabilistic metric (or distance) which takes values $F_{x, y}(t)$ identified with a probability distance density function $H$ : $\mathbf{R} \rightarrow[0,1]$ in the set of all the distance distribution functions $\mathbf{L}$.

A Menger PM-space is a triplet $(X, F, \Delta)$, where $(X, F)$ is a PM-space which satisfies:

$$
\begin{aligned}
& F_{x, y}\left(t_{1}+t_{2}\right) \geq \Delta\left(F_{x, z}\left(t_{1}\right), F_{z, y}\left(t_{2}\right)\right) ; \quad \forall x, y, z \in X, \\
& \forall t_{1}, t_{2} \in \mathbf{R}_{0+}
\end{aligned}
$$

under $\Delta:[0,1] \times[0,1] \rightarrow[0,1]$ is a $t$-norm (or triangular norm) belonging to the set $\mathbf{T}$ of $t$-norms which satisfies the properties:

1. $\Delta(a, 1)=a$

2. $\Delta(a, b)=\Delta(b, a)$

3. $\Delta(c, d) \geq \Delta(a, b)$ if $c \geq a, d \geq b$

4. $\Delta(\Delta(a, b), c)=\Delta(a, \Delta(b, c))$

A property which follows from the above ones is $\Delta(a, 0)=0$ for $a \in[0,1]$. Typical continuous $t$-norms are the minimum $t$-norm defined by $\Delta_{M}(a, b)=\min (a, b)$, the product $t$-norm defined by $\Delta_{P}(a, b)=a . b$ and the Lukasiewicz (or nilpotent-minimum) $t$-norm defined by $\Delta_{L}(a, b)=\max (a+b-1,0)$ which are related by the inequalities $\Delta_{L} \leq \Delta_{P} \leq \Delta_{M}$.

The (probabilistic) diameter of a subset $A$ of $X$ is a function from $\mathbf{R}_{0+}$ to $[0,1]$ defined by $D_{A}(z)=$ $\sup _{t<z} \inf _{x, y \in A} F_{x, y}(t)$ and $A$ is probabilistically bounded if $D_{A}^{p}=$ sup $D_{A}(z)=1 \quad\left(D_{A}^{p} \quad\right.$ can be defined equivalently as $z \in \mathbf{R}_{+}$

$\left.\lim _{z \rightarrow \infty} D_{A}(z)\right)$, probabilistically semibounded if $0<D_{A}^{p}<1$ and probabilistically unbounded if $D_{A}^{p}=0[1,2]$. The diameter of a subset $A \subset X$ in the PM-space $(X, F)$, induced by a metric space $(X, d)$, refers to maximum real interval measure, where the argument of the probabilistic metric is unity, that is,

$$
\operatorname{diam} A=\left\{\inf \left\{t \in \mathbf{R}_{+}:\left(\sup _{x, y \in A}\left(F_{x, y}(t): d(x, y)=\sup _{x, y \in A} d(x, y)\right)\right)=1\right\} \quad \text { if }\left\{t \in \mathbf{R}_{+}:\left(\sup _{x, y \in A}\left(F_{x, y}(t): d(x, y)=\sup _{x, y \in A} d(x, y)\right)\right)=1\right\} \neq \emptyset\right.
$$


Example 1.1 Let $X$ be an abstract nonempty set, $(X, F)$ be a PM-space and $(X, d)$ be a companion metric space and let $A$ be a nonempty subset of $X$ with $F_{x, y}(t)=\frac{\alpha(x, y) t}{\beta(x, y) t+d(x, y)}$ for $t \leq t_{1}$, where $t_{1}=\frac{\sup _{x, y \in A} d(x, y)}{\bar{\alpha}-\bar{\beta}}$, and $F_{x, y}(t)=1$ for $t>t_{1}$ with some given positive real functions subject to $\beta(x, y)=$ $\bar{\beta}, \alpha(x, y)=\bar{\alpha}(\geq \bar{\beta}) \in \mathbf{R}_{+} \quad$ and $\quad \alpha(x, y)=\beta(x, y)=1 \quad$ if $d(x, y)<\sup _{x, y \in A} d(x, y)$. In this case, $\operatorname{diam}(A)=t_{1} \leq \infty$ being, in particular, infinity if $\bar{\alpha}=\bar{\beta}$ (i.e., the probability one is reached as a limit as $t \rightarrow \infty)$ or if $\sup _{x, y \in A} d(x, y)$ is arbitrarily large (i.e., if $A$ is unbounded as a subset of the metric space $(X, d))$. If $\sup _{x, y \in A} d(x, y)<\infty$ and $\bar{\alpha}>\bar{\beta}$ then $\operatorname{diam}(A)<\infty$.

The (probabilistic) distance in-between the subsets $A$ and $B$ of $X$ defines the argument interval length of zero probability distance in-between points of two subsets $A$ and $B$ of $X$ and it is defined as:

$D=d(A, B)=\inf \left(z \in \mathbf{R}_{0+}: \sup _{x \in A, y \in B} F_{x, y}(z)=0\right)$

Definition 1.1 $[7,8,16]$ Let $(X, F, \Delta)$ be a Menger PMspace. Then:

1. A sequence $\left\{x_{n}\right\}$ in $X$ is said to be convergent to $x$ in $X$ if, for every $\varepsilon, \lambda \in \mathbf{R}_{+}$, there exists $n_{0}=n_{0}(\varepsilon, \lambda) \in$ $\mathbf{Z}_{0+}$ such that $F_{x_{n}, x}(\varepsilon)>1-\lambda$, whenever $n \geq n_{0}$.

2. A sequence $\left\{x_{n}\right\}$ in $X$ is said to be a Cauchy sequence if, for every $\varepsilon, \lambda \in \mathbf{R}_{+}$, there exists $n_{0}=n_{0}(\varepsilon, \lambda) \in$ $\mathbf{Z}_{0+}$ such that $F_{x_{n}, x_{m}}(\varepsilon)>1-\lambda$, whenever $n, m \geq n_{0}$.

3. $(X, F, \Delta)$ is complete if every Cauchy sequence in $X$ is convergent to a point in $X$.

4. A sequence $\left\{x_{n}\right\}$ is said to be $G$-Cauchy if, for every $\varepsilon \in \mathbf{R}_{+}, \lim _{n \rightarrow \infty} F_{x_{n}, x_{n+m}}(\varepsilon)=1, \forall m \in \mathbf{Z}_{0+}$.

5. $(X, F, \Delta)$ is $G$-complete if every $G$-Cauchy sequence in $X$ is convergent in $X$.

Assertion 1.1 Let $(X, F, \Delta)$ be a Menger PM-space with $\Delta=\Delta_{M}, \Delta_{P}$ or $\Delta_{L}$. The following properties hold:

1. $\left\{x_{n}\right\} \subset X$ convergent $\Rightarrow\left\{x_{n}\right\}$ is Cauchy $\Rightarrow\left\{x_{n}\right\}$ is $G$-Cauchy.

2. $(X, F, \Delta) G$-complete $\Rightarrow(X, F, \Delta)$ is complete

Proof Proof of (i) Step 1 We first prove that $\left\{x_{n}\right\}$ convergent $\Rightarrow\left\{x_{n}\right\}$ is Cauchy. Since $\left\{x_{n}\right\}$ is convergent then for every $\varepsilon, \lambda \in(0,1) \in \mathbf{R}_{+}$, there exists $n_{0}=$ $n_{0}(\varepsilon, \lambda) \in \mathbf{Z}_{0+} \quad$ such that $F_{x_{m}, x}(\varepsilon / 2)>1-\lambda / 2, \quad \forall n$, $m\left(\geq n_{0}\right) \in \mathbf{Z}_{0+}$. Then, since $F: \mathbf{R} \rightarrow[0,1]$ is non-decreasing, one gets:

$$
\begin{aligned}
F_{x_{n}, x_{m}}(\varepsilon) \geq & \Delta\left(F_{x_{n}, x}(\varepsilon / 2), F_{x_{m}, x}(\varepsilon / 2)\right) \\
\geq & \min \left(\Delta_{M}\left(F_{x_{n}, x}(\varepsilon / 2), F_{x_{m}, x}(\varepsilon / 2)\right),\right. \\
& \Delta_{P}\left(F_{x_{n}, x}(\varepsilon / 2), F_{x_{m}, x}(\varepsilon / 2)\right), \\
& \Delta_{L}\left(F_{x_{n}, x}(\varepsilon / 2), F_{x_{m}, x}(\varepsilon / 2)\right) \\
= & \min \left(\min \left(F_{x_{n}, x}(\varepsilon / 2), F_{x_{m}, x}(\varepsilon / 2)\right),\right. \\
& F_{x_{n}, x}(\varepsilon / 2) \cdot F_{x_{m}, x}(\varepsilon / 2), \\
& \left.\max \left(F_{x_{n}, x}(\varepsilon / 2)+F_{x_{m}, x}(\varepsilon / 2)-1\right), 0\right) \\
= & F_{x_{n}, x}(\varepsilon / 2)+F_{x_{m}, x}(\varepsilon / 2)-1 \\
= & \Delta_{L}\left(F_{x_{n}, x}(\varepsilon / 2), F_{x_{m}, x}(\varepsilon / 2)\right) \\
> & 2(1-\lambda / 2)-1=1-\lambda ; \quad \forall n, m\left(\geq n_{0}\right) \in \mathbf{Z}_{0+}
\end{aligned}
$$

and then $\left\{x_{n}\right\}$ is a Cauchy. Since the above inequalities hold for any $n, m\left(\geq n_{0}\right) \in \mathbf{Z}_{0+}$, it turns out that $\liminf _{n \rightarrow \infty} F_{x_{n}, x_{n+m}}(\varepsilon)>1-\lambda, \quad \forall m \in \mathbf{Z}_{+}$.

Proof of (i) Step 2 We next prove by contradiction that $\left\{x_{n}\right\}$ is Cauchy $\Rightarrow\left\{x_{n}\right\}$ is G-Cauchy. Assume that $\left\{x_{n}\right\}$ is Cauchy while it is not $G$-Cauchy. Then, $\lim _{n \rightarrow \infty} \inf _{x_{n}, x_{n+m}}\left(\varepsilon_{1} / 2\right)>1-\lambda \quad$ and $\lim _{n \rightarrow \infty} \sup _{x_{n}, x_{n+m}}\left(\varepsilon_{1}\right)<1-2 \lambda$, for some $m \in \mathbf{Z}_{0+}$, some $\varepsilon_{1} \in \mathbf{R}_{+}$and some given $\lambda=\lambda\left(\varepsilon_{1}\right) \in(0,1 / 2)$. Since $F_{x, y}(t)$ is non-decreasing in $t$ for all $x, y \in X$, so that $F_{x, y}\left(\varepsilon_{1}\right) \geq F_{x, y}\left(\varepsilon_{1} / 2\right)$, then:

$$
\begin{aligned}
& 1-2 \lambda>\liminf _{n \rightarrow \infty} F_{x_{n}, x_{n+m}}\left(\varepsilon_{1}\right) \geq \liminf _{n \rightarrow \infty} F_{x_{n}, x_{n+m}}\left(\varepsilon_{1} / 2\right)>1 \\
& \quad-\lambda,
\end{aligned}
$$

$\forall m \in \mathbf{Z}_{+}$. But then $\lambda<\lambda / 2$. Then, $\left\{x_{n}\right\}$ is $G$-Cauchy.

Proof of (ii): Let $(X, F, \Delta)$ be $G$-complete and let $\left\{x_{n}\right\} \subset X$ be any given Cauchy sequence. Then $\left\{x_{n}\right\}$ is $G$-Cauchy, from property (1), and convergent to some $x \in X$ since $(X, F, \Delta)$ is $G$-complete. Since $\left\{x_{n}\right\}$ is an arbitrary Cauchy sequence convergent in $X$, it turns out that $(X, F, \Delta)$ is complete.

The $(\varepsilon, \lambda)$-topology in a Menger in a PM-space $(X, F, \Delta)$ is a Hausdorff topology introduced by the family of neighborhoods $N_{x}$ of a point $x \in x$ given by $N_{x}=$ $\left\{N_{x}(\varepsilon, \lambda): \varepsilon \in \mathbf{R}_{+}, \lambda \in(0,1)\right\} \quad$ where $N_{x}(\varepsilon, \lambda)=\left\{z \in X: F_{x, z}(\varepsilon)>1-\lambda\right\}$. In this topology, a function is continuous at $x_{0} \in X$ if and only if $\left\{f\left(x_{n}\right)\right\} \rightarrow$ $f\left(x_{0}\right)$ for every convergent sequence $\left\{x_{n}\right\} \rightarrow x_{0}$. See $[1,16]$ for more details.

We next denote by $\varphi\left(z^{+}\right)$and $\varphi\left(z^{-}\right)$, respectively, the right and left limits of $\varphi(t)$ as $t \rightarrow z$.

Definition 1.2 A function $\varphi: \mathbf{R} \rightarrow \mathbf{R}_{0+}$ is said to be a $\Phi_{x y}$-function if, for given real constants $x, y \in \mathbf{R}_{0+}$, with $y \geq x$, it satisfies the following conditions: 
1. $\varphi(t)$ is strictly increasing for $t \in[x, \infty)$

2. $\varphi\left(x^{+}\right)=y$

3. $\varphi(t)$ is everywhere left-continuous

4. $\varphi(t)=0$ for $t \in(-\infty, x]$

The set of functions $\Phi_{x x}$ is simply denoted by $\Phi_{x}$. If $\varphi: \mathbf{R} \rightarrow \mathbf{R}_{0+}$ is in $\Phi_{x}$ then $\varphi(x)=\varphi\left(x^{-}\right)=0 \leq \varphi\left(x^{+}\right)=x$ and then if $\varphi \in \Phi_{0}$, it is continuous at $t=0$. Note also that the particular set of functions $\Phi_{0}$ coincides with the set of functions $\Phi$ of $[15,16]$ which have continuity at cero. Definition 1.2 will be used in the following to establish the class of contractions under investigation using functions in the sets $\Phi_{D}$ and $\Phi_{D \hat{D}}$, where $D$ is the distance in-between adjacent subsets of the cyclic disposal in $X$.

Definition 1.3 [16] A function $\psi: \mathbf{R}_{0+} \rightarrow \mathbf{R}_{0+}$ is said to be a $\Psi$-function if it continuous with $\psi(0)=0$ and $\psi^{n}\left(a_{n}\right) \rightarrow 0$ when $a_{n} \rightarrow 0$ as $n \rightarrow \infty$.

\section{Main results on best proximity points for $p$-cyclic $\alpha-\psi$-type contractions}

The definition of a $p(\geq 2)$-cyclic $\alpha$ - $\psi$-type contraction follows:

Definition 2.1 Let $(X, F)$ be a PM-space and let $A_{i}$ be nonempty subsets of $X, \forall i \in \bar{p}$ such that $D=d\left(A_{i}, A_{i+1}\right)$ is the common distance in-between adjacent subsets, $\forall i \in \bar{p}$. Then $T: \bigcup_{i \in \bar{p}} A_{i} \rightarrow \bigcup_{i \in \bar{p}} A_{i}$ is a $p(\geq 2)$-cyclic $\alpha$ - $\psi$-type contraction if $T\left(A_{i}\right) \subseteq A_{i+1}, \forall i \in \bar{p}$ and there exist two functions $\alpha:\left(\bigcup_{i \in \bar{p}} A_{i}\right) \times\left(\bigcup_{i \in \bar{p}} A_{i}\right) \times \mathbf{R}_{+} \rightarrow \mathbf{R}_{0+} \quad$ and $\psi \in \Psi$ satisfying the following inequality:

$$
\begin{aligned}
& \alpha(x, y, t)\left(\frac{1}{F_{T x, T y}(\varphi(K t+(1-K) D))}-1\right) \\
& \quad \leq \psi\left(\frac{1}{F_{x, y}(\varphi(t))}-1\right)
\end{aligned}
$$

$\forall(x, y) \in A_{i} \times A_{i+1}, \forall i \in \bar{p}, \forall t(>D) \in \mathbf{R}_{+}$,

where $K \in(0,1)$, for any given $\varphi \in \Phi_{D \hat{D}}$ and any given real $\hat{D} \geq D$.

Note that if $T: \bigcup_{i \in \bar{p}} A_{i} \rightarrow \bigcup_{i \in \bar{p}} A_{i}$ is $p(\geq 2)$-cyclic and if $x \in A_{i}$ for some $i \in \bar{p}$ then for any $j \in \bar{p}$ and $n \in \mathbf{Z}_{0+}$, $T^{n p+i+j} x \in A_{n p+i+j}=A_{k}$ for some $k \in \bar{p}$ since if $n, m \in \mathbf{Z}_{+}$ and $n \equiv m(\bmod p)$ then $A_{m}=A_{n}$. In particular, $A_{i+n p}=A_{i}, \forall i \in \bar{p}, \forall n \in \mathbf{Z}_{0+}$. It can be pointed out that $p$ cyclic contractions include the case of cyclic self-mappings $T$ on $X$ such that $X=\bigcup_{i \in \bar{p}} A_{i}$. In this case, $\left\{A_{i}\right\}_{i \in \bar{p}}$ is said to be a cyclic representation of $(X, T)$. On the other hand, note that is an $\alpha-\psi$-type contraction if (2.1) holds with $D=0$ for $t \in \mathbf{R}_{+}$[16]. The distances in-between adjacent subsets are assumed to be identical just to facilitate the exposition by simplifying the contractive condition to the form (2.1) so as to make less involved their associate calculations. Note that the distances inbetween adjacent subsets in non-expansive cyclic selfmappings are identical in uniformly convex Banach spaces [27].

An equivalent constraint to (2.1) is now discussed:

Proposition 2.1 The constraint (2.1) is identical to:

$$
\begin{aligned}
& F_{T x, T y}(\varphi(t)) \\
& \quad \geq \frac{\alpha\left(x, y, K^{-1}(t-D)+D\right)}{\alpha\left(x, y, K^{-1}(t-D)+D\right)+\psi\left(F_{x, y}^{-1}\left(\varphi\left(K^{-1}(t-D)+D\right)\right)-1\right)},
\end{aligned}
$$

$\forall(x, y) \in A_{i} \times A_{i+1}, \forall i \in \bar{p}, \forall t(>D) \in \mathbf{R}_{+}$which is also identical, if $\alpha(x, y, t) \neq 0, \quad \forall(x, y) \in A_{i} \times A_{i+1}, \quad \forall i \in \bar{p}$, $\forall t(>D) \in \mathbf{R}_{+}$, to

$$
\begin{gathered}
F_{T x, T y}^{-1}(\varphi(t)) \leq 1+\alpha^{-1}\left(x, y, K^{-1}(t-D)+D\right) \\
\psi\left(F_{x, y}^{-1}\left(\varphi\left(K^{-1}(t-D)+D\right)\right)-1\right)
\end{gathered}
$$

Proof Note that, given $K \in(0,1)$ and $D \geq 0$, the function $\beta_{K, D}:[D, \infty) \rightarrow[D, \infty) \quad$ defined by $\beta_{K, D}(t)=K t+$ $(1-K) D$ for $t \in(D, \infty)$ is a strictly increasing, bijective and bicontinuous function of (then continuous) inverse $\beta_{K, D}^{-1}(t)=K^{-1}(t-D)+D$ for $t \in(D, \infty)$, which can be extended by continuity to $t=D$ by defining $\beta(D)=D$.Then, one gets from (2.1) that

$$
\frac{F_{T x, T y}(\varphi(t))}{1-F_{T x, T y}(\varphi(t))} \geq \frac{\alpha\left(x, y, K^{-1}(t-D)+D\right)}{\psi\left(F_{x, y}^{-1}\left(\varphi\left(K^{-1}(t-D)+D\right)\right)-1\right)},
$$

$\forall(x, y) \in A_{i} \times A_{i+1}, \forall i \in \bar{p}, \forall t(>D) \in \mathbf{R}_{+}$which is identical to (2.2). Equation (2.3) follows directly from (2.1) if $\alpha\left(x, y, K^{-1}(t-D)+D\right) \neq 0$.

Note from (2.2) that, if $\alpha(x, y, t)=1$ and $\varphi(t)=\psi(t)=t, \forall x, y \in X, \forall t \in \mathbf{R}$, then the $p$-cyclic $\alpha-\psi$ type contraction $T: \bigcup_{i \in \bar{p}} A_{i} \rightarrow \bigcup_{i \in \bar{p}} A_{i}$ becomes a $p$-cyclic $B$-contraction since one gets $F_{T x, T y}(t) \geq F_{x, y}\left(K^{-1}(t-D)\right.$ $+D), \forall x, y \in X, \forall t \in \mathbf{R}_{+}$from (2.2) if $\alpha(x, y, t)=1$ and $\varphi(t)=\psi(t)=t$. Thus, a $p$-cyclic $B$-contraction is a particular type of $p$-cyclic $\alpha-\psi$-type contraction. See [2] for the case $D=0$. Some basic properties of a $p$-cyclic $\alpha-\psi$-type contraction are now given:

Proposition 2.2 Let $(X, F)$ be a PM-space. Let $T$ : $\bigcup_{i \in \bar{p}} A_{i} \rightarrow \bigcup_{i \in \bar{p}} A_{i}$ be a p-cyclic $\alpha$ - $\psi$-type contraction with $A_{i} \subset X$ being bounded, $\forall i \in \bar{p}$ with $D=d\left(A_{i}, A_{i+1}\right), \forall i \in \bar{p}$ and $\bar{D}=\max _{i \in \bar{p}} \operatorname{diam}\left(A_{i}\right)$ being the distance in-between adjacent subsets, $\forall i \in \bar{p}$. Then, the following properties hold provided that $\varphi \in \Phi_{D \hat{D}}$ for any given $\hat{D} \geq D$ : 
1. $F_{T^{n} x, T^{n} y}(\varphi(t))=1, \quad \forall(x, y) \in A_{i} \times A_{i+1}, \quad \forall i \in \bar{p}$, $\forall t(>D+2 \bar{D}) \in \mathbf{R}_{+}, \forall n \in \mathbf{Z}_{0+}$.

2. $\lim _{t \rightarrow \infty} F_{T^{n} x, T^{n} y}(\varphi(t))=1, \quad \forall(x, y) \in A_{i} \times A_{i+1}, \quad \forall i \in \bar{p}$, $\forall n \in \mathbf{Z}_{0+}$.

3. $F_{x, y}(\varphi(t))=F_{x, y}(\tau)=0, \forall(x, y) \in A_{i} \times A_{i+1}, \quad \forall i \in \bar{p}$, $t \in(-\infty, D), \tau \in(-\infty, \hat{D})$.

4. $F_{x, y}\left(\varphi\left(D^{+}\right)\right)=F_{x, y}(\hat{D})=0 ; \quad \forall(x, y) \quad \in A_{i} \times$ $A_{i+1}, \quad \forall i \in \bar{p} \quad$ if $\hat{D}=D, F_{x, y}\left(\varphi\left(D^{+}\right)^{+}\right)=F_{x, y}\left(\hat{D}^{+}\right)$ $=1 ; \quad \forall(x, y) \in A_{i} \times A_{i+1}, \quad \forall i \in \bar{p} \quad$ if $\hat{D}=$ $D, F_{x, y}\left(\varphi\left(D^{+}\right)\right)=F_{x, y}(\hat{D})=1$;

$\forall(x, y) \in A_{i} \times A_{i+1}, \quad \forall i \in \bar{p} \quad$ if $\hat{D}>D$

where $\hat{D}=\varphi\left(D^{+}\right)=\lim _{t \rightarrow D^{+}} \varphi(t)$ and $F_{x, y}\left(\hat{D}^{+}\right)=\lim _{\tau \rightarrow D^{+}}$ $\lim _{t \rightarrow \tau^{+}} F_{x, y}(\varphi(t))$.

Proof Since $A_{i}$ and $A_{i+1}$ are bounded then the maximum distance in-between any two points of adjacent subsets is not larger than $D+2 \bar{D}$. Then, $\lim _{s \rightarrow \infty} F_{x, y}(s)=\max _{i \in \bar{p}}$ $\max _{z \in A_{i}, \omega \in A_{i+1}} F_{z, \omega}(\varphi(t))=1, \forall(x, y) \in A_{i} \times A_{i+1}, \forall i \in \bar{p}$, if $t \in(D+2 \bar{D}, \infty)$, since the distance distribution function $F: \mathbf{R} \rightarrow[0,1]$ is non-decreasing and left-continuous, and $T\left(A_{i}\right) \subseteq A_{i+1}$ with $D=d\left(A_{i}, A_{i+1}\right), \forall i \in \bar{p}$. Then,

$F_{x, y}^{-1}\left(\varphi\left(K^{-1}(t-D)+D\right)\right)=F_{x, y}\left(\varphi\left(K^{-1}(t-D)+D\right)\right)$

$=1$ for $t \in(D+2 \bar{D}, \infty)$ so that

$\psi\left(F_{x, y}^{-1}\left(\varphi\left(K^{-1}(t-D)+D\right)\right)-1\right)$

$=\psi(0)=0, \quad t \in(D+2 \bar{D}, \infty)$

since $\quad K \in(0,1), \quad K^{-1}(t-D)+D>2 \bar{D}+D \quad$ and $\varphi\left(K^{-1}(t-D)+D\right)>\varphi(2 \bar{D}+D) \forall t(>D+2 \bar{D}) \in \mathbf{R}_{+}$, since $\varphi: \mathbf{R} \rightarrow \mathbf{R}_{0+}$ is strictly increasing and left-continuous in $(D, \infty), F: \mathbf{R} \rightarrow[0,1]$ is non-decreasing and left continuous and $\psi(0)=0$. Thus, from Proposition 2.1 [Eq. (2.3)], $F_{T x, T y}(\varphi(t))=F_{T x, T y}^{-1}(\varphi(t))=1$ for $t \in(D+$ $2 \bar{D}, \infty)$. Again, since $K \in(0,1), \quad K^{-1}(t-D)+$ $D>2 \bar{D}+D$ and $\varphi\left(K^{-1}(t-D)+D\right)>\varphi(2 \bar{D}+D)$, since $\varphi: \mathbf{R} \rightarrow \mathbf{R}_{0+}$ is strictly increasing and left-continuous in $(D, \infty)$, if $\varphi \in \Phi_{D}$ (Definitions 2.1 and 1.2), $F: \mathbf{R} \rightarrow[0,1]$ is non-decreasing and left continuous and $\psi(0)=0$, then one gets for $t \in(D+2 \bar{D}, \infty)$, that:

$$
\begin{gathered}
F_{T x, T y}\left(\varphi\left(K^{-1}(t-D)+D\right)\right)=F_{T x, T y}^{-1}\left(\varphi\left(K^{-1}(t-D)+D\right)\right) \\
=1 ; \\
\psi\left(F_{T x, T y}^{-1}\left(\varphi\left(K^{-1}(t-D)+D\right)\right)-1\right)=\psi(0)=0
\end{gathered}
$$

so that, again one gets from Proposition 2.1 [Eq. (2.3)], $F_{T^{2} x, T^{2} y}(\varphi(t))=F_{T^{2} x, T^{2} y}^{-1}(\varphi(t))=1$ for $t \in(D+2 \bar{D}, \infty)$. Now, proceed by complete induction by assuming that $F_{T^{n} x, T^{n} y}(\varphi(t))=F_{T^{n} x, T^{n} y}^{-1}(\varphi(t))=1$ for $t \in(D+2 \bar{D}, \infty)$ for any given $n \in \mathbf{Z}_{0+}$. Since $K \in(0,1), K^{-1}(t-D)+$ $D>2 \bar{D} \quad+D, \varphi\left(K^{-1}(t-D)+D\right)>\varphi(2 \bar{D}+D)$ for $t \in(D+2 \bar{D}, \infty), F: \mathbf{R} \rightarrow[0,1]$ is non-decreasing and left continuous and $\psi(0)=0$, one has for $t \in(D+2 \bar{D}, \infty), T^{n} x \subset A_{i+n} \equiv A_{j}$ and $T^{n} y \subset A_{i+n+1} \equiv$ $A_{j+1}$ for a unique integer $j=j(i) \in \overline{p-1} \cup\{0\}$ fulfilling $n=q p+j-i \geq i$ for some $q=q(i) \in \mathbf{Z}_{+}$and the given $i \in \bar{p}$ that:

$$
\begin{aligned}
& F_{T^{n} x, T^{n} y}\left(\varphi\left(K^{-1}(t-D)+D\right)\right) \\
& \quad=F_{T^{n} x, T^{n} y}^{-1}\left(\varphi\left(K^{-1}(t-D)+D\right)\right)=1 ; \\
& \quad \psi\left(F_{T^{n} x, T^{n} y}^{-1}\left(\varphi\left(K^{-1}(t-D)+D\right)\right)-1\right)=\psi(0)=0 .
\end{aligned}
$$

Then, from Proposition 2.1 [Eq. (2.3)], $F_{T^{n+1}} x, T^{n+1} y$ $(\varphi(t))=F_{T^{n+1} x, T^{n+1} y}^{-1}(\varphi(t))=1$ for $t \in(D+2 \bar{D}, \infty)$. Hence, the proofs of Properties (1) and (2) follow by complete induction.

Properties (3)-(5) follow directly from the definitions of the sets $\Phi_{D \hat{D}}$ for $\tau=\varphi(t)$, being equivalent to $t=t(\tau)=\arg \left(z \in \mathbf{R}: \varphi\left(z^{-}\right)=\tau\right.$ for $\left.\tau \in(\varphi(D), \hat{D})\right)$ which is point-wise unique for any $\tau \in(\varphi(D), \hat{D})$, zero for $t \in \mathbf{R}_{0-}$ if $\Phi_{D \hat{D}}$ and, left-continuous and strictly increasing for $t(\in \mathbf{R})>D$.

It turns out that Proposition 2.2 holds, in particular, for $\varphi \in \Phi_{D}$. The $\alpha$-admissibility of $\alpha-\psi$-type contractions is defined to state layer on the main result:

Definitions 2.2 Let $(X, F)$ a PM-space. Then:

1. an $\alpha$ - $\psi$-type contraction $T: X \rightarrow X$ is $\alpha$-admissible for a given function $\alpha: X \times X \times \mathbf{R}_{+} \rightarrow \mathbf{R}_{0+}$ [16] if

$$
\begin{aligned}
\forall x, y & \in X, \forall t \\
& \in \mathbf{R}_{+}[(\alpha(x, y, t) \geq 1) \Rightarrow(\alpha(T x, T y, t) \geq 1)],
\end{aligned}
$$

2. a $p$-cyclic $\alpha$ - $\psi$-type contraction $T: \bigcup_{i \in \bar{p}} A_{i} \rightarrow \bigcup_{i \in \bar{p}} A_{i}$ is $\alpha$-admissible for a given function $\alpha:\left(\bigcup_{i \in \bar{p}} A_{i}\right) \times$

$$
\begin{aligned}
& \left(\bigcup_{i \in \bar{p}} A_{i}\right) \times \mathbf{R}_{+} \rightarrow \mathbf{R}_{0+} \text { if } \\
& \begin{aligned}
\forall(x, y) & \in A_{i} \times A_{i+1}, \forall i \in \bar{p}, \forall t \\
& \in \mathbf{R}_{+}[(\alpha(x, y, t) \geq 1) \Rightarrow(\alpha(T x, T y, t) \geq 1)] .
\end{aligned}
\end{aligned}
$$

Definitions 2.3 $T: \bigcup_{i \in \bar{p}} A_{i} \rightarrow \bigcup_{i \in \bar{p}} A_{i}$ be a $\alpha-\psi$-type $p$ cyclic mapping, $\varphi \in \Phi_{D \hat{D}}$ and $\psi \in \Psi$. Then:

1. the pair $(x, T x) \in\left(\operatorname{cl} A_{i}, \operatorname{cl} A_{i+1}\right)$, where $\operatorname{cl}($.$) stands for$ the closure of the (.)-set, for any given $i \in \bar{p}$ is a pair of quasi-best proximity points if $F_{x, T x}(\hat{D}+\varepsilon)>1-\lambda$ for any given $\varepsilon \in \mathbf{R}_{+}$and $F_{x, T x}(D)=F_{x, T x}\left(D^{-}\right)=0$. 
Each of them is a quasi-best proximity point in the corresponding subset $A_{i}$.

2. A quasi-best proximity point is a best proximity point if $F_{x, T x}(D+\varepsilon)>1-\lambda$ for any given $\varepsilon \in \mathbf{R}_{+}$and $\lambda \in(0,1)$.

Remarks 2.1 Since $\varphi \in \Phi_{D \hat{D}}$ then $\hat{D}=\varphi\left(D^{+}\right) \geq D$. If $\varphi \in \Phi_{D}$, then $\hat{D}=\varphi\left(D^{+}\right)=D$ and, if the pair $(x, T x)$ is a pair of quasi-best proximity points, then $F_{x, T x}(D)=$ $F_{x, T x}\left(\varphi\left(D^{+}\right)\right)=0$ and $F_{x, T x}\left(D^{+}\right)=1$ [see also the two first properties of Proposition 2.2 (iv)].

If $\varphi \in \Phi_{D \hat{D}} \quad$ with $\quad \hat{D}=\varphi\left(D^{+}\right)>D \quad$ then $F_{x, T x}(\hat{D}+\varepsilon)>1-\lambda$ for arbitrarily small positive real constants $\varepsilon$ and $\lambda$ so that $F_{x, T x}\left(\hat{D}^{+}\right)=1$ and $F_{x, T x}(D)=$ $F_{x, T x}\left(D^{-}\right)=0$ but it is not guaranteed that $F_{x, T x}\left(D^{+}\right)=1$, then, it is not guaranteed in this case that $(x, T x)$ is a pair of best proximity points if $\varphi$ is not continuous at $D$.

The most important of the main results of this paper follows below:

Theorem 2.1 Let $(X, F, \Delta)$ be a G-complete PM-space and let $T: \bigcup_{i \in \bar{p}} A_{i} \rightarrow \bigcup_{i \in \bar{p}} A_{i}$ be a $p$-cyclic $\alpha-\psi$-type contraction with $\varphi \in \Phi_{D \hat{D}}$ for some $\hat{D}(\in \mathbf{R}) \geq D$ satisfying the following conditions:

1. $D=d\left(A_{i}, A_{i+1}\right)>0$ and $\operatorname{diam}\left(A_{i}\right)>\hat{D}-D, \forall i \in \bar{p}$,

2. $T$ is $\alpha$-admissible,

3. there exists $x_{0} \in \bigcup_{i \in \bar{p}} A_{i}$ such that $\alpha\left(x_{0}, T x_{0}, t\right) \geq 1$, $\forall t \in \mathbf{R}_{+}$,

4. if $\left\{x_{n}\right\}(\subset X) \rightarrow x$ is a Picard iteration generated as $x_{n+1}=T x_{n}, \forall n \in \mathbf{Z}_{0+}$ with $x_{0} \in \bigcup_{i \in \bar{p}} A_{i}$, such that $\alpha\left(x_{n}, T x_{n}, t\right) \geq 1, \quad \forall n \in \mathbf{Z}_{0+}, \quad \forall t \in \mathbf{R}_{+} \quad$ then $\alpha\left(x_{n}, x, t\right) \geq 1, \forall n \in \mathbf{Z}_{0+}, \forall t \in \mathbf{R}_{+}$.

Then, the following properties hold:

1. $F_{T^{n p} x_{0}, T^{(n+m) p+j} x_{0}}((p m+j)(\varphi(D)+\varepsilon))>1-\lambda$, $\forall t(>D) \in \mathbf{R}_{+}, \forall j \in \bar{p}$ for any given real constants $\varepsilon \in \mathbf{R}_{+}$and $\lambda \in(0,1)$, some $n_{0}=n_{0}(\varepsilon, \lambda) \in \mathbf{Z}_{+}$and $\forall n\left(\geq n_{0}\right) \in \mathbf{Z}_{0+}, \forall m \in \mathbf{Z}_{+}$, and

$$
\begin{aligned}
& F_{T^{n} x_{0}, T^{n+1} x_{0}}(\varphi(t)) \\
& \quad \geq \frac{1}{1+\psi^{n}\left(F_{x_{0}, T x_{0}}^{-1}\left(\varphi\left(K^{-n}(t-D)+D\right)\right)-1\right)}, \\
& \forall t(>D) \in \mathbf{R}_{+}, \quad \forall n \in \mathbf{Z}_{+} .
\end{aligned}
$$

2. If $\varphi \in \Phi_{0}$ and $\bigcap_{i \in \bar{p}} A_{i} \neq \emptyset$ then

$$
\begin{aligned}
& \lim _{n \rightarrow \infty} F_{T^{n} x_{0}, T^{n+1} x_{0}}(t)=\lim _{n \rightarrow \infty} F_{T^{n p+j} x_{0}, T^{(n+m) p+j+1} x_{0}}(t)=1, \\
& \quad \forall t \in \mathbf{R}_{+} .
\end{aligned}
$$

Furthermore, $\left\{T^{n} x_{0}\right\} \subset \bigcup_{i \in \bar{p}} A_{i}$ and $\left\{T^{n p+j} x_{0}\right\} \subset A_{i+j}$ are both Cauchy and $G$-Cauchy convergent sequences to a limit point $x \in \bigcap_{i \in \bar{p}} c l A_{i}$. If the subsets $A_{i}$ are closed for $i \in \bar{p}$ then $x=T x=T^{p} x$, that is, it is a fixed point of the self-mappings $T: \bigcup_{i \in \bar{p}} A_{i} \rightarrow \bigcup_{i \in \bar{p}} A_{i}$ and $T^{p}\left(\equiv T \circ T^{p-1}\right): \bigcup_{i \in \bar{p}} A_{i} \mid A_{k} \rightarrow A_{k}, \forall k \in \bar{p}, \forall x_{0} \in A_{i}$, $\forall i \in \bar{p}, \forall j \in \overline{p-1} \cup\{0\}$.

3. If $A_{i}$ is closed, $\forall i \in \bar{p}$ and $\bigcap_{i \in \bar{p}} A_{i}=\emptyset$ then if $x_{0} \in A_{i}$ for any $i \in \bar{p}$ then there is a limit cycle $\left(\bar{x}_{i}, T \bar{x}_{i}, \ldots, T^{p-1} \bar{x}_{i}\right)$, to which the sequence $\left\{T^{n} x_{0}\right\}$ converges, with $T^{p} \bar{x}_{i}=\bar{x}_{i}, \bar{x}_{i} \in A_{i}, \bar{x}_{i+j}=T^{j} \bar{x}_{i} \in A_{i+j}$, $\forall i \in \bar{p}, \forall j \in \overline{p-1} \cup\{0\}$ being a fixed point of the composite self-mappings (of domain and image restricted to each of the subsets) $T^{p}: \bigcup_{i \in \bar{p}} A_{i} \mid A_{k} \rightarrow A_{k}, \forall k \in \bar{p}$, and it is also a quasibest proximity point (in particular, a best proximity point if $\varphi \in \Phi_{D} \equiv \Phi_{D \hat{D}}$ ) of $T: \bigcup_{i \in \bar{p}} A_{i} \rightarrow \bigcup_{i \in \bar{p}} A_{i}$. The subsequence $\left\{T^{n p+j} x_{0}\right\}$ in $A_{i+j}$ converges to $x_{i+j}=T^{j} x_{i}, \forall j \in \bar{p}$ if $x_{0} \in A_{i}$ for some $i \in \bar{p}$. Furthermore:

$$
\begin{aligned}
& \lim _{n \rightarrow \infty} F_{T^{n p} x_{0}, T^{(n+m) p} x_{0}}(\varepsilon)=F_{x_{0}, T x_{0}}(\infty)=1, \\
& \quad F_{T^{n p} x_{0}, T^{(n+m) p} x_{0}}(\varepsilon)>1-\lambda \\
& F_{T^{n p j} x_{0}, T^{n p j+1} x_{0}}(\varphi(D)+\varepsilon)>1-\lambda, \\
& \quad \lim _{n \rightarrow \infty} F_{T^{n p j} x_{0}, T^{n p j+1} x_{0}}(\varphi(D)+\varepsilon)=F_{\bar{x}_{i+j}, \bar{x}_{i+j+1}}=1
\end{aligned}
$$

for any given $\varepsilon \in \mathbf{R}_{+}, \lambda \in(0,1)$ and for some $n_{0}=n_{0}(\varepsilon, \lambda), \forall n\left(\geq n_{0}\right) \in \mathbf{Z}_{0+}$.

Proof Let $x_{0} \in \bigcup_{i \in \bar{p}} A_{i}$ such that the condition (3) holds. Since $T: \bigcup_{i \in \bar{p}} A_{i} \rightarrow \bigcup_{i \in \bar{p}} A_{i}$ is a $p$-cyclic $\alpha$ - $\psi$-type contraction, $\quad x_{0} \in \bigcup_{i \in \bar{p}} A_{i}, \quad \alpha\left(x_{0}, T x_{0}, t\right) \geq 1$, $x_{n+1}=T x_{n}=T^{n+1} x_{0} \neq x_{n}, \forall n \in \mathbf{Z}_{0+}, \forall t \in \mathbf{R}_{+}$and $T$ is $\alpha-$ admissible, assume that $\quad \alpha\left(x_{j}, T x_{j}, t\right) \geq 1$, $\forall j \in \bar{n} \cup\{0\} \in \mathbf{Z}_{0+}, \quad \forall t \in \mathbf{R}_{+}$. Since $\alpha\left(x_{n}, T x_{n}, t\right) \geq 1 \Rightarrow \alpha\left(T x_{n}, T x_{n+1}, t\right) \geq 1, \quad \forall t \in \mathbf{R}_{+}$, then $\alpha\left(x_{j}, T x_{j}, t\right) \geq 1, \forall j \in \overline{n+1} \cup\{0\} \in \mathbf{Z}_{0+}, \forall t \in \mathbf{R}_{+}$. Then, $\alpha\left(x_{j}, T x_{j}, t\right) \geq 1, \quad \forall j \in \bar{n} \cup\{0\} \in \mathbf{Z}_{0+}, \quad \forall t \in \mathbf{R}_{+} \quad \Rightarrow$ $\alpha\left(x_{j}, T x_{j}, t\right) \geq 1, \forall j \in \overline{n+1} \cup\{0\} \in \mathbf{Z}_{0+}, \forall t \in \mathbf{R}_{+}$. It has been proved by complete induction that $\alpha\left(x_{n}, T x_{n}, t\right) \geq 1$, $\forall n \in \mathbf{Z}_{0+}, \quad \forall t \in \mathbf{R}_{+} \quad$ provided that $\alpha\left(x_{0}, T x_{0}, t\right) \geq 1$, $\forall t \in \mathbf{R}_{+}$. Then $\quad \alpha\left(x_{n}, T x_{n}, t\right) \in[1,+\infty) \quad$ and $\alpha^{-1}\left(x_{n}, T x_{n}, t\right) \in(0,1], \quad \forall n \in \mathbf{Z}_{0+}, \quad \forall t \in \mathbf{R}_{+} \quad$ if $\alpha\left(x_{0}, T x_{0}, t\right) \geq 1, \forall t \in \mathbf{R}_{+}$. On the other hand, since $\varphi \in$ $\Phi_{D \hat{D}}$ and since $T: \bigcup_{i \in \bar{p}} A_{i} \rightarrow \bigcup_{i \in \bar{p}} A_{i}$ is a $p$-cyclic $\alpha$ - $\psi$-type contraction, $\varphi$ is strictly increasing in $(D, \infty)$ with $\varphi(D)=\hat{D} \geq D>0$ (see Definitions 2.1 and 1.2) and then 
there exists $t(>D) \in \mathbf{R}_{+}$such that $F_{x_{0}, T x_{0}}(t)>0$, $\forall t(>D) \in \mathbf{R}_{+}$. Since the distance distribution function is non-decreasing and left-continuous and $K^{-1}>1$, then $F_{x_{0}, T x_{0}}\left(\varphi\left(K^{-1}(t-D)+D\right)\right) \geq F_{x_{0}, T x_{0}}(t)>0$ and $0 \leq F_{x_{0}, T x_{0}}^{-1}$ $\left(\varphi\left(K^{-1}(t-D)+D\right)\right)-1<+\infty, \forall t(>D) \in \mathbf{R}_{+}$. Now, note that $\alpha^{-1}\left(x_{n}, T x_{n}, t\right) \in(0,1]$ since $\alpha\left(x_{n}, T x_{n}, t\right)$ $\in[1, \infty) ; \quad \forall n \in \mathbf{Z}_{0+}, \forall t \in \mathbf{R}_{+}$. Thus, if $\alpha^{-1}\left(T^{n} x_{0}\right.$, $\left.T^{n+1} x_{0}, K^{-1}(t-D)+D\right) \in(0,1] ; \forall n \in \mathbf{Z}_{0+}, \forall t(>D) \in$ $\mathbf{R}_{+}$and, furthermore,

Case a $\lim _{n \rightarrow \infty} \inf ^{-1}\left(T^{n} x_{0}, T^{n+1} x_{0}, K^{-1}(t-D)+D\right)$ $>0, \forall t(>D) \in \mathbf{R}_{+}$

then one gets from (2.3) that

$$
\begin{aligned}
& F_{T x_{0}, T^{2} x_{0}}^{-1}(\varphi(t)) \leq 1+\alpha^{-1}\left(x_{0}, T x_{0}, K^{-1}(t-D)+D\right) \\
& \quad \psi\left(F_{x_{0}, T x_{0}}^{-1}\left(\varphi\left(K^{-1}(t-D)+D\right)\right)-1\right) \\
& \leq 1+\psi\left(F_{x_{0}, T x_{0}}^{-1}\left(\varphi\left(K^{-1}(t-D)+D\right)\right)-1\right), \\
& \quad \forall t(>D) \in \mathbf{R}_{+},
\end{aligned}
$$

equivalently,

$$
\begin{aligned}
& F_{T x_{0}, T^{2} x_{0}}^{-1}\left(\varphi\left(K^{-1}(t-D)+D\right)\right) \leq 1 \\
& \quad+\psi\left(F_{x_{0}, T x_{0}}^{-1}\left(\varphi\left(K^{-2}(t-D)+D\right)\right)-1\right), \\
& \quad \forall t(>D) \in \mathbf{R}_{+}
\end{aligned}
$$

and replacing in (2.5) $x_{0} \rightarrow T x_{0}, T x_{0} \rightarrow T^{2} x_{0}$ with the use of (2.6) leads to:

$$
\begin{aligned}
& F_{T^{2} x_{0}, T^{3} x_{0}}^{-1}(\varphi(t)) \leq 1+\psi\left(F_{T x_{0}, T^{2} x_{0}}^{-1}\left(\varphi\left(K^{-1}(t-D)+D\right)\right)-1\right) \\
& \quad \leq 1+\psi^{2}\left(F_{x_{0}, T x_{0}}^{-1}\left(\varphi\left(K^{-2}(t-D)+D\right)\right)-1\right), \quad \forall t(>D) \in \mathbf{R}_{+}
\end{aligned}
$$

and proceeding recursively in the same way:

$$
\begin{aligned}
& F_{T^{n} x_{0}, T^{n+1} x_{0}}^{-1}(\varphi(t)) \leq 1+\psi\left(F_{T^{n-1} x_{0}, T^{n} x_{0}}^{-1}\left(\varphi\left(K^{-1}(t-D)+D\right)\right)-1\right) \\
& \quad \leq 1+\psi^{2}\left(F_{T^{n-2} x_{0}, T^{n-1} x_{0}}^{-1}\left(\varphi\left(K^{-2}(t-D)+D\right)\right)-1\right) \\
& \quad \leq 1+\psi^{n}\left(F_{x_{0}, T x_{0}}^{-1}\left(\varphi\left(K^{-n}(t-D)+D\right)\right)-1\right), \\
& \forall t(>D) \in \mathbf{R}_{+}, \quad \forall n \in \mathbf{Z}_{+},
\end{aligned}
$$

equivalently,

$$
\begin{gathered}
F_{T^{n} x_{0}, T^{n+1} x_{0}}(\varphi(t)) \geq \frac{1}{1+\psi^{n}\left(F_{x_{0}, T x_{0}}^{-1}\left(\varphi\left(K^{-n}(t-D)+D\right)\right)-1\right)}, \\
\forall t(>D) \in \mathbf{R}_{+}, \quad \forall n \in \mathbf{Z}_{+}
\end{gathered}
$$

Since $K^{-1}>1$ and $\varphi \in \Phi_{D \hat{D}}$ then $\lim _{n \rightarrow+\infty}\left(K^{-n}(t-D)\right.$ $+D)=+\infty, \lim _{n \rightarrow \infty} \varphi\left(K^{-n}(t-D)+D\right)=+\infty, \forall t(>D)$ $\in \mathbf{R}_{+}$and $\lim _{n \rightarrow \infty} F_{x_{0}, T x_{0}}^{-1}\left(\varphi\left(K^{-n}(t-D)+D\right)\right)=1, \forall t(>D)$ $\in \mathbf{R}_{+}$. Thus,

$\lim _{n \rightarrow \infty} \psi^{n}\left(F_{x_{0}, T x_{0}}^{-1}\left(\varphi\left(K^{-n}(t-D)+D\right)\right)-1\right)=0$,

$\forall t(>D) \in \mathbf{R}_{+}$

since $\quad \lim _{n \rightarrow \infty} \psi^{n}\left(F_{x_{0}, T x_{0}}^{-1}\left(\varphi\left(K^{-n}(t-D)+D\right)\right)-1\right)=$ $\lim _{n \rightarrow \infty} \psi^{n}\left(t_{n}^{n}\right)=0$ if $\left\{t_{n}\right\} \rightarrow 0$. Then, $\lim _{n \rightarrow \infty} F_{T^{n} x_{0}, T^{n+1} x_{0}}(\varphi(t))$ $=1, \forall t(>D) \in \mathbf{R}_{+}$from $(2.8 \mathrm{~b})$.

Case $b \lim _{n \rightarrow \infty} \alpha^{-1}\left(T^{n} x_{0}, T^{n+1} x_{0}, K^{-1}(t-D)+D\right)=0$, $\forall t(>D) \in \mathbf{R}_{+}$

We first prove that $\lim _{n \rightarrow \infty} \sup \psi^{n}\left(F_{x_{0}, T x_{0}}^{-1}\left(\varphi\left(K^{-n}(t-D)\right.\right.\right.$ $+D))-1)<\infty, \forall t(>D) \in \mathbf{R}_{+}$. The above condition is identical to $\lim _{n \rightarrow \infty} \sup ^{n}\left(F_{x_{0}, T x_{0}}^{-1}\left(\varphi\left(t_{n}\right)\right)-1\right)<\infty$ with $t_{n}=$ $t_{n}(t)=K^{-n}(t-D)+D$ and, since $K<1, K^{-n} \rightarrow \infty$ as $n \rightarrow \infty$ and $\varphi(t)$ is strictly increasing then $\left\{t_{n}\right\} \rightarrow \infty$ and $\varphi\left(t_{n}\right) \rightarrow \infty$ as $n \rightarrow \infty$. Assume that this is not the case so that $\lim _{n \rightarrow \infty} \sup ^{n}\left(F_{x_{0}, T x_{0}}^{-1}\left(\varphi\left(t_{n}\right)\right)-1\right)=\infty$. Then, since the function $\psi: \mathbf{R}_{0+} \rightarrow \mathbf{R}_{0+}$ is everywhere continuous in its definition domain it can only diverge at infinity and then $\lim _{n \rightarrow \infty} F_{x_{0}, T x_{0}}^{-1}\left(\varphi\left(t_{n}\right)\right)=\infty, \quad$ and equivalently, $\lim _{n \rightarrow \infty}$ $F_{x_{0}, T x_{0}}\left(\varphi\left(t_{n}\right)\right)=\lim _{t_{n} \rightarrow \infty} F_{x_{0}, T x_{0}}\left(\varphi\left(t_{n}\right)\right)=0$. But this would lead to the contradiction that $F_{x_{0}, T x_{0}}(t)$ is not non-decreasing. As a result, $\lim _{n \rightarrow \infty} \sup ^{n}\left(F_{x_{0}, T x_{0}}^{-1}\left(\varphi\left(t_{n}\right)\right)-1\right)<\infty$, and then

$$
\begin{aligned}
& \lim _{n \rightarrow \infty}\left(\alpha^{-1}\left(T^{n_{1}} x_{0}, T^{n_{1}+1} x_{0}, K^{-1}(t-D)+D\right)\right. \\
& \left.\psi^{n}\left(F_{x_{0}, T x_{0}}^{-1}\left(\varphi\left(K^{-n}(t-D)+D\right)\right)-1\right)\right)=0
\end{aligned}
$$

leading to $\lim _{n \rightarrow \infty} F_{T^{n} x_{0}, T^{n+1}} \quad x_{0}^{-1}(\varphi(t))=\lim _{n \rightarrow \infty} F_{T^{n} x_{0}, T^{n+1} x_{0}}$ $(\varphi(t))=1, \forall t(\stackrel{\eta \rightarrow \infty}{>} D) \in \mathbf{R}_{+}$from (2.8a).

Since $\hat{D} \geq \varphi\left(D^{+}\right)$, one concludes for Cases a and $\mathrm{b}$ that $\lim _{n \rightarrow \infty} F_{T^{n p j} x_{0}, T^{n p j+1} x_{0}}(\varphi(D)+t)=1, \forall t \in \mathbf{R}_{+}$with $T^{n p j} x_{0} \in$ $A_{i+j}$ and $T^{n p j+1} x_{0} \in A_{i+j+1}, \forall j \in \bar{p}$ if $x_{0} \in A_{i}$ for any given $i \in \bar{p} \operatorname{since} \operatorname{diam}\left(A_{i}\right)>\hat{D}-D, \forall i \in \bar{p}$. Then, for any given real $\varepsilon \in \mathbf{R}_{+}$and $\lambda \in(0,1)$, there is $n_{0}=n_{0}(\varepsilon, \lambda) \in \mathbf{Z}_{0+}$ such that

$F_{T^{n p j} x_{0}, T^{n p j+1} x_{0}}(\varphi(D)+\varepsilon)>1-\lambda, \quad \forall n \geq n_{0}, \quad \forall j \in \bar{p}$,

since the distance distribution function is non-decreasing and left-continuous. On the other hand,

$$
\begin{aligned}
& F_{T^{n p} x_{0}, T^{(n+m) p+j} x_{0}}((p m+j)(\varphi(D)+\varepsilon)) \\
& \quad \geq \Delta_{M}\left(F_{T^{n p} x_{0}, T^{n p+1} x_{0}}(\varphi(D)+\varepsilon), F_{T^{n p+1} x_{0}, T^{n p+2} x_{0}}\right. \\
& \left.\quad(\varphi(D)+\varepsilon), \ldots, F_{T^{(n+m) p+j-1} x_{0}, T^{(n+m) p+j} x_{0}}(\varphi(D)+\varepsilon)\right)>1-\lambda ;
\end{aligned}
$$


$\forall t(>D) \in \mathbf{R}_{+}, \forall j \in \bar{p}$ and, for any given $\varepsilon \in \mathbf{R}_{+}$and $\lambda \in(0,1), \quad F_{T^{n p} x_{0}, T^{(n+m) p+j}} x_{0}((p m+j)(\varphi(D)+\varepsilon))>1-\lambda$ for $n\left(\geq n_{0}\right) \in \mathbf{Z}_{0+}, \forall m \in \mathbf{Z}_{+}$. Property (1) has been proved.

Property (2) relies on the case when $\varphi \in \Phi_{0}$ and $\bigcap_{i \in \bar{p}} A_{i} \neq \emptyset$ since $D=0$. If $\varphi \in \Phi_{0}$ then $D=\hat{D}=$ $\varphi(0)=0$ so that

$$
\begin{aligned}
& F_{T^{n} x_{0}, T^{n+1} x_{0}}(\varepsilon)>1-\lambda, \\
& \quad F_{T^{n p+j} x_{0}, T^{(n+1) p+j} x_{0}}((p m+j) \varepsilon)>1-\lambda, \\
& \quad \forall n\left(\geq n_{0}\right) \in \mathbf{Z}_{0+}, \quad \forall m \in \mathbf{Z}_{+},
\end{aligned}
$$

and $\quad \lim _{n \rightarrow \infty} F_{T^{n} x_{0}, T^{n+1} x_{0}}(t)=\lim _{n \rightarrow \infty} F_{T^{n p+j} x_{0}, T^{(n+m) p+j+1} x_{0}}(t)=1$, $\forall t \in \mathbf{R}_{+}$. Since $(X, F, \Delta)$ is $G$-complete, then $\left\{T^{n p+j} x_{0}\right\} \rightarrow$ $\bar{x}_{i+j}\left(\in \operatorname{cl}\left(A_{i+j}\right)\right), \forall j \in \overline{p-1} \cup\{0\}, \forall x_{0} \in A_{i}$. Assume that $\bar{x}_{j} \neq \bar{x}_{j+k}$ for some $j, k \in \bar{p}$. Since $\left(X, F, \Delta_{M}\right)$ is a Menger PM-space then from (2.10) with $D=\hat{D}=\varphi(0)=0$ :

$$
\begin{aligned}
& F_{T^{n p+j+\ell} x_{0}, T^{n p+j+\ell+1} x_{0}}(\varepsilon / k)>1-\lambda, \quad \forall n\left(\geq n_{0}\right) \in \mathbf{Z}_{0+} ; \\
& \quad \lim _{n \rightarrow \infty} F_{T^{n p+j+\ell} x_{0}, T^{n p+j+\ell+1} x_{0}}(\varepsilon / k)=1,
\end{aligned}
$$

for $\ell \in \overline{k-1} \cup\{0\}$, any $\varepsilon \in \mathbf{R}_{+}$and any real $\lambda \in(0,1)$ what implies that $\lim _{n \rightarrow \infty} F_{T^{n+j} x_{0}, T^{n p+j+k} x_{0}}(t)=1, \forall t \in \mathbf{R}_{+}$and, from the first property of (1.1), $\bar{x}_{j}=\bar{x}_{j+k}$, a contradiction, and then $\bar{x}_{i}=\bar{x}, \forall i \in \bar{p}$. So, the $p$ sequences $\left\{T^{n p+i} x_{0}\right\}, \forall i \in \bar{p}$ have a unique limit point in $\bigcap_{i \in \bar{p}} A_{i}$ provided that such a set is nonempty and from Assertion 1.1 they are Cauchy and $G$ Cauchy sequences and then convergent since $(X, F, \Delta)$ is $G$ complete. In addition, for any $\varepsilon \in \mathbf{R}_{+}$and any real $\lambda \in(0,1)$ there is $n_{01}=n_{01}(\varepsilon, \lambda) \in \mathbf{Z}_{0+}$ such that from (2.2) for $D=0$ since $\left\{T^{n} x_{0}\right\}$ is Cauchy, then $G$-Cauchy, and convergent to $x$, then $\lim _{n \rightarrow \infty} \psi\left(F_{T^{n} x_{0}, x}^{-1}\left(\varphi\left(K^{-1} \varepsilon / 3\right)\right)-1\right)=\psi(0)=1$ and $\lim _{n \rightarrow \infty} F_{T^{n+1} x_{0}, T x}(\varepsilon / 3)=1$ so that

$$
\begin{aligned}
& F_{T^{n+1} x_{0}, T x}(\varepsilon / 3) \\
& \geq \frac{\alpha\left(T^{n} x_{0}, x, K^{-1} \varepsilon / 3\right)}{\alpha\left(T^{n} x_{0}, x, K^{-1} \varepsilon / 3\right)+\psi\left(F_{T^{n} x_{0}, x}^{-1}\left(\varphi\left(K^{-1} \varepsilon / 3\right)\right)-1\right)} \\
& \quad \forall n\left(\in \mathbf{Z}_{0+}\right) \\
& F_{T^{n+1} x_{0}, T x}(\varepsilon / 3)>1-\lambda ; \quad \forall n\left(\geq n_{01}\right) \in \mathbf{Z}_{0+}
\end{aligned}
$$

so that for some $n_{0}=n_{0}(\varepsilon, \lambda)\left(\geq n_{01}\right) \in \mathbf{Z}_{0+}$ and for any arbitrary $\varepsilon \in \mathbf{R}_{+}$and $\lambda \in(0,1)$ using the third and fourth properties of the triangular norms, one gets:

$$
\begin{aligned}
& F_{x, T x}(\varepsilon) \geq \min \left(F_{x, T^{n} x_{0}}(\varepsilon / 3), F_{T^{n+1} x_{0}, T^{n} x_{0}}(\varepsilon / 3),\right. \\
& \left.\quad F_{T^{n+1} x_{0}, T x}(\varepsilon / 3)\right)>1-\lambda, \quad \forall n\left(\geq n_{0}\right) \in \mathbf{Z}_{0+},
\end{aligned}
$$

Thus, $F_{x, T x}\left(0^{+}\right)=1$ so that $x=T x$ from the property 1 of (1.1). By replacing $x \rightarrow T x$ and $T x \rightarrow T^{2} x$ in (2.13), we prove $x=T^{2} x$. Proceeding in the same way, it is proved that $T^{i} x=x, \forall i \in \bar{p}$. So, $x$ is a limit point of $\left\{T^{n p+i} x_{0}\right\}$, $\forall i \in \bar{p}$ and $\left\{T^{n} x_{0}\right\}$ which is also a fixed point of $T$ : $\bigcup_{i \in \bar{p}} A_{i} \rightarrow \bigcup_{i \in \bar{p}} A_{i}$ and $T^{p}: \bigcup_{i \in \bar{p}} A_{i}\left|A_{j} \rightarrow \bigcup_{i \in \bar{p}} A_{i}\right| A_{j}, \forall j \in \bar{p}$ if the subsets $A_{i}$ are closed $\forall i \in \bar{p}$. Hence, Property (2) has been proved.

On the other hand, it follows from (1.2) for Menger PMspaces and the properties of (1.3) for triangular norms for the general case that $\hat{D}=\varphi\left(D^{+}\right) \geq D>0$ since, furthermore, $\Delta_{M}(x, x) \geq x$ for each $x \in[0,1]$, that

$$
\begin{aligned}
& F_{T^{n p} x_{0}, T^{(n+m) p} x_{0}}(t) \geq \Delta_{M}\left(F_{T^{n p} x_{0}, T^{n p+1} x_{0}}((1-K) t), F_{T^{n p+1} x_{0}, T^{(n+m) x_{x_{0}}}}(K t)\right) \\
& \geq \Delta_{M}\left(F_{x_{0}, T x_{0}}\left(\frac{1-K^{m p}}{1-K} K^{-n p}((1-K) t-D)+D\right)\right. \text {, } \\
& \left.\Delta_{M}\left(F_{T^{n p+1} x_{0}, T^{n p+2} x_{0}}(K(1-K) t), F_{T^{n p+2} x_{0}, T^{(n+m) p} x_{0}}\left(K^{2} t\right)\right)\right) \\
& \geq \Delta_{M}\left(F_{x_{0}, T x_{0}}\left(\frac{1-K^{m p}}{1-K} K^{-n p}((1-K) t-D)+D\right)\right. \text {, } \\
& \Delta_{M}\left(F_{x_{0}, T x_{0}}\left(\frac{1-K^{m p}}{1-K} K^{-n p-1}(K(1-K) t-D)+D\right),\right. \\
& \left.\left.F_{T^{n+2} x_{0}, T^{(n+m) p} x_{0}}\left(K^{2} t\right)\right)\right) \\
& =\Delta_{M}\left(F_{x_{0}, T x_{0}}\left(\frac{1-K^{m p}}{1-K} K^{-n p}((1-K) t-D)+D\right),\right. \\
& \Delta_{M}\left(F_{x_{0}, T x_{0}}\left(\frac{1-K^{m p}}{1-K} K^{-n p}((1-K) t-D)+D\right)\right. \\
& \left.\left.F_{T^{n p+2} x_{0}, T^{(n+m) p} x_{0}}\left(K^{2} t\right)\right)\right) \\
& \geq \Delta_{M}\left(F_{x_{0}, T x_{0}}\left(\frac{1-K^{m p}}{1-K} K^{-n p}((1-K) t-D)+D\right),\right. \\
& \Delta_{M}\left(F_{x_{0}, T x_{0}}\left(\frac{1-K^{m p}}{1-K} K^{-n p}((1-K) t-D)+D\right),\right. \\
& \left.\left.F_{T^{(n+m) p-1} x_{0}, T^{(n+m) p} x_{0}}\left(K^{m p-1} t\right)\right)\right) \\
& \geq \Delta_{M}\left(F_{x_{0}, T x_{0}}\left(\frac{1-K^{m p}}{1-K} K^{-n p}((1-K) t-D)+D\right)\right. \text {, } \\
& \Delta_{M}\left(F_{x_{0}, T x_{0}}\left(\frac{1-K^{m p}}{1-K} K^{-n p}((1-K) t-D)+D\right),\right. \\
& \left.\left.F_{x_{0}, T x_{0}}\left(K^{-(n+m) p+1}\left(K^{m p-1} t-D\right)+D\right)\right)\right) \\
& =\Delta_{M}\left(F_{x_{0}, T x_{0}}\left(\frac{1-K^{m p}}{1-K} K^{-n p}((1-K) t-D)+D\right)\right. \text {, } \\
& \Delta_{M}\left(F_{x_{0}, T x_{0}}\left(\frac{1-K^{m p}}{1-K} K^{-n p}((1-K) t-D)+D\right)\right. \text {, } \\
& \left.\left.F_{x_{0}, T x_{0}}\left(\frac{1-K^{m p}}{1-K} K^{-n p}((1-K) t-D)+D\right)\right)\right) \\
& \geq \Delta_{M}\left(F_{x_{0}, T x_{0}}\left(\frac{1-K^{m p}}{1-K} K^{-n p}((1-K) t-D)+D\right),\right. \\
& \left.F_{x_{0}, T x_{0}}\left(\frac{1-K^{m p}}{1-K} K^{-n p}((1-K) t-D)+D\right)\right) \\
& \geq F_{x_{0}, T x_{0}}\left(\frac{1-K^{m p}}{1-K} K^{-n p}((1-K) t-D)+D\right) ; \quad \forall t \in \mathbf{R}_{+}, \\
& \forall m \in \mathbf{Z}_{0+}, \quad \forall n \in \mathbf{Z}_{+}
\end{aligned}
$$

Then, one gets for any given $t, \lambda(<1) \in \mathbf{R}_{+}$, some $n_{0}=n_{0}(t, \lambda)$ and $\forall n \geq n_{0}$. 


$$
\begin{aligned}
& \lim _{n \rightarrow \infty} F_{T^{n p} x_{0}, T^{(n+m) p} x_{0}}(t)=F_{x_{0}, T x_{0}}(\infty)=1 ; \\
& \quad F_{T^{n p} x_{0}, T^{(n+m) x_{x_{0}}}}(t)>1-\lambda,
\end{aligned}
$$

$\forall x_{0} \in \bigcup_{i \in \bar{p}} A_{i}, \forall m \in \mathbf{Z}_{+}$since $F_{x_{0}, T x_{0}}(t)$ is non-decreasing with supremum over $t \in \mathbf{R}_{+}$equalizing unity. Thus, $\left\{T^{n p+j} x_{0}\right\} \rightarrow \bar{x}_{i+j}\left(\in \operatorname{cl}\left(A_{i+j}\right)\right), \quad \forall j \in \overline{p-1} \cup\{0\}, \forall x_{0} \in$ $A_{i}, \forall i \in \bar{p}$. Since $D>0$ then $\bar{x}_{i} \neq \bar{x}_{j}, \forall i, j(\neq i) \in \bar{p}$. In addition, either

$$
\begin{aligned}
& F_{\bar{x}_{i+j}, T^{P} \bar{x}_{i+j}}(\varepsilon) \geq \min \left(F_{\bar{x}_{i+j}, T^{n p+j} x_{0}}(\varepsilon / 4), F_{T^{n p+j} x_{0}, T^{(n+1) p+j} x_{0}}(\varepsilon / 4),\right. \\
& \left.F_{T^{(n+1) p+j} x_{0}, \bar{x}_{i+j}}(\varepsilon / 4), F_{\bar{x}_{i+j}, T^{p} \bar{x}_{i+j}}(\varepsilon / 4)\right) \\
\geq & \min \left(F_{\bar{x}_{i+j}, T^{n p+j} x_{0}}(\varepsilon / 4), F_{T^{n p+j} x_{0}, T^{(n+1) p+x_{0}}}(\varepsilon / 4),\right. \\
& \left.F_{T^{(n+1) p+j} x_{0}, \bar{x}_{i+j}}(\varepsilon / 4)\right)>1-\lambda,
\end{aligned}
$$

$\forall n\left(\geq n_{0}\right) \in \mathbf{Z}_{0+}$, or $F_{\bar{x}_{i+j}, T^{P} \bar{x}_{i+j}}(\varepsilon / 4) \leq 1-\lambda$, then either $F_{\bar{x}_{i+j}, T^{P} \bar{x}_{i+j}}(\varepsilon) \leq 1-\lambda, \forall n\left(\geq n_{0}\right) \in \mathbf{Z}_{0+}$ and

$$
\begin{aligned}
& 1-\lambda \geq F_{\bar{x}_{i+j}, T^{n p+j} x_{0}}(\varepsilon) \geq \min \left(F_{\bar{x}_{i+j}, T^{p} \bar{x}_{i+j}}(\varepsilon / 4),\right. \\
& \left.\quad F_{T^{n p+j} x_{0}, T^{(n+1) p+j} x_{0}}(\varepsilon / 4), F_{T^{(n+1) p+j} x_{0}, \bar{x}_{i+j}}(\varepsilon / 4), F_{\bar{x}_{i+j}, T^{p} \bar{x}_{i+j}}(\varepsilon / 4)\right) \\
& \quad \geq \min \left(F_{\bar{x}_{i+j}, T^{n p+j} x_{0}}(\varepsilon / 4), F_{T^{n p+j} x_{0}, T^{(n+1) p+j} x_{0}}(\varepsilon / 4),\right. \\
& \left.F_{T^{(n+1) p+j} x_{0}, \bar{x}_{i+j}}(\varepsilon / 4)\right)>1-\lambda
\end{aligned}
$$

for some $n\left(\geq n_{0}\right) \in \mathbf{Z}_{0+}$, which is a contradiction, or $F_{\bar{x}_{i+j}, T^{T} \bar{x}_{i+j}}(\varepsilon)>1-\lambda$. As a result (2.16) leads, in any case, to (2.17.a) and $F_{\bar{x}_{i+j}, T^{P} \bar{x}_{i+j}}(\varepsilon)>1-\lambda, \forall n\left(\geq n_{0}\right) \in \mathbf{Z}_{0+}$. Thus, $\bar{x}_{j}$ is a fixed point of the composite self-mapping $T^{p}: \bigcup_{i \in \bar{p}} A_{i} \mid A_{j} \rightarrow A_{j}, \forall j \in \bar{p}$. To prove that the fixed points of the composite self-mapping are quasi-best proximity points of the $p$-cyclic $\alpha$ - $\psi$-type contraction $T: \bigcup_{i \in \bar{p}} A_{i} \rightarrow \bigcup_{i \in \bar{p}} A_{i}$, we proceed by contradiction. Assume that this is not the case, so that there is a pair $\left(\bar{x}_{i}, T \bar{x}_{i}\right)$ for some $i \in \bar{p}$ such that there exist $\varepsilon \in \mathbf{R}_{+}, \lambda \in$ $(0,1)$ and a sequence $\left\{n_{k}\right\} \subset \mathbf{Z}_{0+}$ for some $n_{0}=$ $n_{0}(\varepsilon, \lambda) \in \mathbf{Z}_{0+}$ such that, for any $j \in \bar{p}$, one gets that

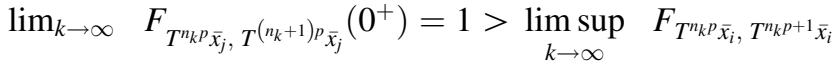
$\left(\hat{D}^{+}\right)$. Since $\left(X, F, \Delta_{M}\right)$ is a Menger space and, since any triangular norm is associative and commutative and since $\Delta_{M}(x, x) \geq x$, one gets the contradiction:

$F_{\bar{x}_{i}, T^{p} \bar{x}_{i}}(\varepsilon) \geq \min _{j \in \bar{p}}\left(F_{\bar{x}_{i}, T^{j} \bar{x}_{i}}\right)=q(1-\lambda) \geq 1-\lambda$

for some real $q=q(\varepsilon, \lambda) \in(0,1)$ to $\bar{x}_{k}$ being a fixed point of $T^{p}: \bigcup_{i \in \bar{p}} A_{i} \mid A_{k} \rightarrow A_{k}$ for $k \in \bar{p}$. Property (3) has been proved.
Note that if the image of $\alpha(x, y, t)$ is extended to be in $c l \mathbf{R}_{0+}$ (i.e., $\alpha(x, y, t)$ can take also values at $\{+\infty\}$ ), then the proof of Property (1) of Theorem 2.1 is valid with a slight extension by considering also the case that $\alpha^{-1}\left(T^{n_{1}} x_{0}, T^{n_{1}+1} x_{0}, K^{-1}(t-D)+D\right)=0$ for some finite $n_{1} \in \mathbf{Z}_{0+}$ and $\forall t(>D) \in \mathbf{R}_{+}$. Since $\psi \in \Psi$, it is continuous, and then $\psi^{n_{1}}\left(F_{x_{0}, T x_{0}}^{-1}\left(\varphi\left(K^{-n_{1}}(t-D)+D\right)\right)-1\right)<\infty$ for any finite $n_{1} \in \mathbf{Z}_{+}$so that

$$
\begin{aligned}
& \alpha^{-1}\left(T^{n_{1}-1} x_{0}, T^{n_{1}} x_{0}, K^{-1}(t-D)+D\right) \psi^{n_{1}} \\
& \quad\left(F_{x_{0}, T x_{0}}^{-1}\left(\varphi\left(K^{-n_{1}}(t-D)+D\right)\right)-1\right)=0, \quad \forall t(>D) \in \mathbf{R}_{+} .
\end{aligned}
$$

Then, we could use a similar recursive procedure as that used for the case up till the $\left(n_{1}-1\right)$-iteration, since Proposition 2.1 remains valid, see Eqs. (2.2) and (2.3), so that:

$$
\begin{aligned}
& F_{T^{n_{1}} x_{0}, T^{n_{1}+1} x_{0}}(\varphi(t)) \leq 1+\alpha^{-1}\left(T^{n_{1}-1} x_{0}, T^{n_{1}} x_{0}, K^{-1}(t-D)\right. \\
& \quad+D) \psi^{n_{1}}\left(F_{x_{0}, T x_{0}}^{-1}\left(\varphi\left(K^{-n_{1}}(t-D)+D\right)\right)-1\right)=1
\end{aligned}
$$

Note that Theorem 2.1 generalizes some results on fixed points given in $[7,8,15,16,28]$ for either non-cyclic selfmappings or cyclic self-mappings on union of sets which intersect to quasi-best proximity points and best proximity points in the case that such sets do not intersect. On the other hand, a direct consequence of Theorem 2.1 is the following corollary for the case that $\varphi \in \Phi_{D}$. The results are based on the fact that $\varphi\left(D^{-}\right)=D$ and $\varphi(t)=0$ if $t \in$ $[0, D]$ and $\varphi \in \Phi_{D}$ while it generalizes results on fixed points for the cases of either non-cyclic self-mappings or cyclic self-mappings with nonempty intersections of the involved subsets obtained in $[7,8,15,16,28]$ :

Corollary 2.1 Let $(X, F, \Delta)$ be a G-complete Menger $P M$-space and $T: \bigcup_{i \in \bar{p}} A_{i} \rightarrow \bigcup_{i \in \bar{p}} A_{i}$ be a p-cyclic $\alpha-\psi$ type contraction satisfying the following conditions:

1. $D=d\left(A_{i}, A_{i+1}\right)>0, \quad \forall i \in \bar{p}$,

2. $T$ is $\alpha$-admissible,

3. there exists $x_{0} \in \bigcup_{i \in \bar{p}} A_{i}$ such that $\alpha\left(x_{0}, T x_{0}, t\right) \geq 1$, $\forall t \in \mathbf{R}_{+}$,

4. if $\left\{x_{n}\right\}(\subset X) \rightarrow x$ is a Picard iteration generated as $x_{n+1}=T x_{n}, \forall n \in \mathbf{Z}_{0+}$ with $x_{0} \in \bigcup_{i \in \bar{p}} A_{i}$, such that $\alpha\left(x_{n}, T x_{n}, t\right) \geq 1, \forall n \in \mathbf{Z}_{0+}, \forall t \in \mathbf{R}_{+}$then $\alpha\left(x_{n}, x, t\right)$ $\geq 1, \forall n \in \mathbf{Z}_{0+}, \forall t \in \mathbf{R}_{+}$.

Then, the following properties hold:

1. If $\varphi \in \Phi_{0}$ and $\bigcap_{i \in \bar{p}} A_{i} \neq \emptyset$ then $\left\{T^{n} x_{0}\right\} \subset \bigcup_{i \in \bar{p}} A_{i}$ and $\left\{T^{n p+j} x_{0}\right\} \subset A_{i+j}$ are both Cauchy and G-Cauchy convergent sequences to a limit point $x \in \bigcap_{i \in \bar{p}} \operatorname{cl} A_{i}$, with cl $A_{i}$ being the closure of $A_{i}$. If $A_{i}$ are closed for 
$i \in \bar{p}$ then $x=T x=T^{p} x$, that is, it is a fixed point of the self-mappings $T: \bigcup_{i \in \bar{p}} A_{i} \rightarrow \bigcup_{i \in \bar{p}} A_{i} \quad$ and $T^{p}\left(\equiv T \circ T^{p-1}\right): \bigcup_{i \in \bar{p}} A_{i} \mid A_{k} \rightarrow A_{k}, \forall k \in \bar{p}, \forall x_{0} \in A_{i}$, $\forall i \in \bar{p}, \forall j \in \overline{p-1} \cup\{0\}$.

2. If $\varphi \in \Phi_{D}, A_{i}$ is closed $\forall i \in \bar{p}$ and $\bigcap_{i \in \bar{p}} A_{i}=\emptyset$ then, if $x_{0} \in A_{i}$ for any $i \in \bar{p}$, there is a limit cycle $\left(\bar{x}_{i}, T \bar{x}_{i}, \ldots, T^{p-1} \bar{x}_{i}\right)$, to which the sequence $\left\{T^{n} x_{0}\right\}$ converges, with $T^{p} \bar{x}_{i}=\bar{x}_{i}, \bar{x}_{i} \in A_{i}, \bar{x}_{i+j}=T^{j} \bar{x}_{i} \in A_{i+j}$, $\forall i \in \bar{p}, \forall j \in \overline{p-1} \cup\{0\}$ being a fixed point of the composite self-mapping $T^{p}: \bigcup_{i \in \bar{p}} A_{i} \mid A_{k} \rightarrow A_{k}, \forall k \in \bar{p}$, and being also a best proximity point of $T: \bigcup_{i \in \bar{p}} A_{i} \rightarrow \bigcup_{i \in \bar{p}} A_{i}$. The subsequence $\left\{T^{n p+j} x_{0}\right\}$ in $A_{i+j}$ converges to $x_{i+j}=T^{j} x_{i}, \forall j \in \bar{p}$ if $x_{0} \in A_{i}$ for some $i \in \bar{p}$. Furthermore,

$$
\begin{aligned}
& \lim _{n \rightarrow \infty} F_{T^{n p} x_{0}, T^{(n+m) p} x_{0}}(\varepsilon)=F_{x_{0}, T x_{0}}(\infty)=1, \\
& \quad F_{T^{n p} x_{0}, T^{(n+m) p} x_{0}}(\varepsilon)>1-\lambda \\
& F_{T^{n p j} x_{0}, T^{n p j+1} x_{0}}(\varphi(D)+\varepsilon)>1-\lambda, \\
& \quad \lim _{n \rightarrow \infty} F_{T^{n p j} x_{0}, T^{n p j+1} x_{0}}(\varphi(D)+\varepsilon)=F_{\bar{x}_{i+j}, \bar{x}_{i+j+1}}=1
\end{aligned}
$$

for any given $\varepsilon \in \mathbf{R}_{+}, \lambda \in(0,1) \cap \mathbf{R}$ and some $n_{0}=n_{0}(\varepsilon, \lambda), \forall n\left(\geq n_{0}\right) \in \mathbf{Z}_{0+}$.

The following result is an extended version of a parallel result given in [16] for uniqueness of fixed points of (noncyclic) self-mappings of a $p$-cyclic $\alpha-\psi$-type contraction on $X$. The result is concerned with (a) the uniqueness of best proximity points of $p$-cyclic $\alpha$ - $\psi$-type contractions, being corresponding fixed points of the composite self-mappings restricted to each subset of the cyclic disposal, and (b) their confluence to a unique fixed point of the $p$-cyclic $\alpha-\psi$-type contraction if the subsets intersect.

Theorem 2.2 Assume all the hypotheses of Corollary 2.1 and the additional one which follow:

(5) For all $x, y \quad \in \bigcup_{i \in \bar{p}} c l A_{i}$, there is $z \in \quad \bigcup_{i \in \bar{p}} c l A_{i}$ such that $\min (\alpha(x, z, t), \alpha(y, z, t)) \geq 1, \quad \forall t(>D) \in$ $\mathbf{R}_{+}$. Then, there is $\bar{x}_{i} \in c l A_{i}$, such that $\bar{x}_{i} \in A_{i}$ if $A_{i}$ is closed, $\forall i \in \bar{p}$, which is the unique best proximity point of $T: \bigcup_{i \in \bar{p}} A_{i} \rightarrow \bigcup_{i \in \bar{p}} A_{i}$ and the unique fixed point of $T^{p}: \bigcup_{i \in \bar{p}} A_{i} \mid A_{k} \rightarrow A_{k}, \forall k \in \bar{p}$. If $\varphi \in \Phi_{0}$ with $D=0$ and $\bigcap_{i \in \bar{p}} A_{i}$ is nonempty and closed then there is a unique fixed point of $T: \bigcup_{i \in \bar{p}} A_{i} \rightarrow \bigcup_{i \in \bar{p}} A_{i}$ and of the composite selfmappings $T^{p}: \bigcup_{i \in \bar{p}} A_{i} \mid A_{j} \rightarrow A_{j}, \forall j \in \bar{p}$.
Proof Let $u \in c l A_{i}, v \in c l A_{j}$ be best proximity points for any given $i, j \in \bar{k} \quad$ such that $u=T^{n p} u=T^{p} u$ and $v=T^{n p} v=T^{p} v, \forall n \in \mathbf{Z}_{+}$so that there is $z \in \bigcup_{i \in \bar{p}} c l A_{i}$ such that $\min (\alpha(u, z, t), \alpha(v, z, t)) \geq 1, \forall t(>D) \in \mathbf{R}_{+}$.

Thus, there exist integers $m=m(i, u, z) \in \overline{p-1} \cup\{0\}$ and $\ell=\ell(j, v, z) \in \overline{p-1} \cup\{0\}$, such that $T^{p n+m} z \in A_{i+1}$ and $T^{p n+\ell} z \in A_{j+1}$, and since $T$ is $\alpha$-admissible and $\min (\alpha(x, z, t), \alpha(y, z, t)) \geq 1, \forall t(>D) \in \mathbf{R}_{+}$, one gets:

$$
\begin{aligned}
& \frac{1}{F_{u, T^{p n+m} z}(\varphi(t))}-1 \leq \sup _{n \in \mathbf{Z}_{0+}}\left[\alpha^{-1}\left(u, T^{n p+m} z, t\right)\right] \\
& \psi^{n p-1}\left(\frac{1}{F_{u, T^{m} z}\left(\varphi\left(K^{-(n p+m)}(t-D)+D\right)\right)}-1\right), \\
& \forall t(>D) \in \mathbf{R}_{+}
\end{aligned}
$$

$$
\begin{aligned}
& \frac{1}{F_{v, T^{n+\ell} z}(\varphi(t))}-1 \leq \alpha^{-1}(v, z, t) \psi^{n p-1} \\
& \quad\left(\frac{1}{F_{v, T^{\ell} z}\left(\varphi\left(K^{-(n p+\ell)}(t-D)+D\right)\right)}-1\right), \quad \forall t(>D) \in \mathbf{R}_{+} .
\end{aligned}
$$

Note that $\psi^{n p-1}\left(a_{n p-1}(t, m, u, z)\right) \rightarrow 0$ as $a_{n p-1}(t, m$, $u, z)=\frac{1}{F_{u, T^{m}}\left(\varphi\left(K^{-(n p+m)}(t-D)+D\right)\right)}-1 \rightarrow 0$ as $n \rightarrow \infty$ for all $t(>D) \in \mathbf{R}_{+}$which holds since $F_{u, T^{m} z}\left(\varphi\left(K^{-n p}(t-D)\right.\right.$ $+D)) \rightarrow 1$ as $n \rightarrow \infty$ for all $t(>D) \in \mathbf{R}_{+}$since $\left(K^{-n p}(t-D)+D\right) \rightarrow \infty$ for all $t(>D) \in \mathbf{R}_{+}$as $n \rightarrow \infty$ and since $\varphi(t)>D$ from (2.21), from the properties $\varphi(t)=$ 0 for $t<D, \varphi(D) \geq D$, and $\varphi(t)>D$ for $t>D$ since $\varphi$ : $\mathbf{R} \rightarrow \mathbf{R}_{0+}$ is strictly increasing in $[D, \infty)$. In the same way, note that $\psi^{n p-1}\left(b_{n p-1}(t, m, v, z)\right) \rightarrow 0$ as $b_{n p-1}(t, m, v, z)=$ $\frac{1}{F_{u, T^{m_{z}}\left(\varphi\left(K^{-(n p+m)}(t-D)+D\right)\right)}}-1 \rightarrow 0 \quad$ as $n \rightarrow \infty$ for all $t(>D) \in \mathbf{R}_{+}$. As a result:

$F_{u, T^{p+m} z}(t)=F_{v, T^{p+\ell} z}(t)=1, \quad \forall t(>D) \in \mathbf{R}_{+}$

$F_{u, T^{n+m} z}(t)=F_{v, T^{p+\ell} z}(t)=0, \quad \forall t(\leq D) \in \mathbf{R}_{+}$

with $u \in A_{i}, v \in A_{j}, T^{p n+m} z \in A_{i+1}, T^{p n+\ell} z \in A_{j+1}$ for any $i, j \in \bar{p}$. Thus, if $i=j$ then $u, v \in c l A_{i}, \quad \ell=m$ and $T^{p n+m} z \in c l A_{i+1}$. Assume that $u \neq v$ are best proximity points in $c l A_{i}$, then $T u, T v \in A_{i+1}$ are corresponding adjacent best proximity points and $\left\{T^{p n+m} z\right\} \rightarrow T u$ and $\left\{T^{p n+m} z\right\} \rightarrow T v$. Thus, $T u=T v$ and $u=T^{p} u=T^{p} v=v$ which contradicts $u \neq v$. Since $i \in \bar{p}$ is arbitrary then the set of adjacent best proximity points is unique.

In the particular case that $D=0$, one gets that $u=v$ is a unique fixed point of $T \rightarrow \bigcup_{i \in \bar{p}} A_{i} \rightarrow \bigcup_{i \in \bar{p}} A_{i}$, allocated in the nonempty set $\bigcap_{i \in \bar{p}} c l A_{i}$, and also a fixed point of each 
restricted composite self-mapping $T^{p}: \bigcup_{i \in \bar{p}} A_{i} \mid A_{j} \rightarrow$ $A_{j}, \forall j \in \bar{p}$ since Eqs. (2.23) and (2.24) result to be for $i=j$ :

$F_{u, T^{p n+m} z}(t)=F_{v, T^{p+m_{z}}}(t)=1, \quad \forall t \in \mathbf{R}_{+}$

Example 2.1 Consider real intervals $A=-B=$ $\{z \in \mathbf{R}: z \geq D / 2\} \subset \mathbf{R}$ so that $(\mathbf{R}, d)$ is a complete metric space under some metric $d: X \times X \rightarrow \mathbf{R}_{0+}$ and $D=d(A, B)$. The sequence $\left\{x_{n}\right\} \subset A \cup B$ is generated by 2-cyclic self-mapping $T$ on $A \cup B$ for some real constant $K \in[0,1)$ and any given initial $x_{0} \in \mathbf{R} \equiv A \cup B$, by

$x_{n+1}=-K x_{n}-(1-K)(D / 2) \operatorname{sgn}\left(x_{n}\right), \quad n \in \mathbf{Z}_{+}$

with the extended definition $\operatorname{sgn}(x)=0$ if $x=0$ for the case that $D=0$. The above sequence has the two following subsequences, in $A$ and $B$ if $x_{0} \in A$, respectively, in $B$ and $A$ if $x_{0} \in B$ :

$x_{2 n}=T^{2 n} x_{0}=K^{2 n} x_{0}+\left(1-K^{2 n}\right)(D / 2) \operatorname{sgn}\left(x_{0}\right), \quad n \in \mathbf{Z}_{+}$,
$D=0$ and then a unique fixed point of $T: A \cup B \rightarrow A \cup B$ and $T^{2}: A \cup B \rightarrow A \cup B$. Let $\mathbf{D}_{D}^{+}$be the set of all generalized distance distribution functions of elements defined by $H_{D}(t)=0$ if $t \leq D$ and $H(t)=1$ if $t>D$. Now, define $\varphi(t)=\psi(t)=t$, and:

- a probability mapping $F: \mathbf{R} \times \mathbf{R} \rightarrow \mathbf{D}_{D}^{+}, \quad F_{x, y}(t)=$ $\frac{g t^{p}+D}{g t^{p}+d(x, y)}$ if $t\left(\in \mathbf{R}_{+}\right)>D$ if $x \in A$ and $y \in B$ or $x \in B$ and $y \in A ; F_{x, y}(t)=\frac{g t^{p}}{g t^{p}+d(x, y)}$ if $t\left(\in \mathbf{R}_{+}\right)$if $x, y \in A$ or $x, y \in B ; \quad F_{x, y}(t)=0 \quad$ if $\quad t(\in \mathbf{R}) \leq D \quad$ for $\quad$ all $(x, y) \in A \times B \cup B \times A$, for any given $g, p \in \mathbf{R}_{+}$

- a weighting function $\alpha: \mathbf{R} \times \mathbf{R} \times \mathbf{R}_{+} \rightarrow \mathbf{R}_{0+}$ by $\alpha(x, y, t)=\frac{\delta t^{q}+d(x, y)}{t+\lambda D}$ for some given real constants $\lambda \in$ $(0,1]$ and $\delta \geq 1$.

Note that $\left(\mathbf{R}, F, \Delta_{M}\right)$ is a $G$-complete generalized Menger PM-space since the distance distribution function is a generalized one, [12]. It follows from Proposition 2.1 that

$$
\begin{aligned}
& F_{T x_{n+1}, T x_{n}}(t)=F_{T^{2} x_{n}, T x_{n}}(t)=\frac{g t^{p}+D}{g t^{p}+d\left(T^{2} x_{n}, T x_{n}\right)} \\
& \geq \frac{g t^{p}+D}{g t^{p}+D+K\left(d\left(T x_{n}, x_{n}\right)-D\right)}=\frac{1}{1+\left(g t^{p}+D\right)^{-1} K\left(d\left(T x_{n}, x_{n}\right)-D\right)} \\
& \geq \frac{1}{1+\alpha^{-1}\left(x_{n}, T x_{n}, K^{-1}(t-D)+D\right)\left(g t^{p}+D\right)^{-1} K\left(F_{x_{n}, T x_{n}}^{-1}\left(K^{-1}(t-D)+D\right)-1\right)\left(g\left(K^{-1}(t-D)+D\right)^{p}+D\right)} \\
& \geq \frac{1}{1+\left(F_{x_{n}, T x_{n}}^{-1}\left(K^{-1}(t-D)+D\right)-1\right)} \geq F_{x_{n}, T x_{n}}\left(K^{-1}(t-D)+D\right), \quad n \in \mathbf{Z}_{0+}, \quad \forall t(>D) \in \mathbf{R}_{+}
\end{aligned}
$$

$$
\begin{aligned}
x_{2 n+1}= & T x_{2 n}=T^{2 n+1} x_{0}=-K^{2 n+1} x_{0} \\
& -\left(1-K^{2 n}\right)(D / 2) \operatorname{sgn}\left(x_{0}\right), \quad n \in \mathbf{Z}_{+} .
\end{aligned}
$$

Note that $T: A \cup B \rightarrow A \cup B$ is a 2-cyclic contraction, with the metric being the Euclidean norm, since

$$
\begin{aligned}
& d\left(x_{n+2}, x_{n+1}\right)=\left|x_{n+2}-x_{n+1}\right|=\mid K\left(x_{n}-x_{n+1}\right) \\
& \quad+(1-K) D \operatorname{sgn}\left(x_{n}\right) \mid \leq K d\left(x_{n+2}, x_{n+1}\right) \\
& \quad+(1-K) D, \quad n \in \mathbf{Z}_{0+},
\end{aligned}
$$

and it turns out that $\left\{x_{2 n}\right\} \rightarrow D / 2,\left\{x_{2 n+1}\right\} \rightarrow-D / 2$ if $x_{0} \in A$ and $\left\{x_{2 n}\right\} \rightarrow-D / 2,\left\{x_{2 n+1}\right\} \rightarrow D / 2$ if $x_{0} \in B$ and $x= \pm D / 2$ are the unique best proximity points in $A$ and $B$, respectively, and unique fixed points of $T^{2}: A \rightarrow A$ and, respectively, of $T^{2}: B \rightarrow B$, which are confluent at $x=0$ if since $\quad \alpha^{-1}\left(x_{n}, T x_{n}, K^{-1}(t-D)+D\right) \leq 1, \quad n \in \mathbf{Z}_{0+}$, $\forall t(>D) \in \mathbf{R}_{+}$. Then, note that $T$ is $\alpha$-admissible and since

$$
\begin{aligned}
& d\left(x_{n}, T x_{n}\right)-D=\left(F_{x_{n}, T x_{n}}^{-1}\left(K^{-1}(t-D)+D-1\right)\right) \\
& \quad\left(g\left(K^{-1}(t-D)+D\right)^{p}+D\right) \\
& =\left(F_{x_{n}, T x_{n}}^{-1} K^{-1}(t-D)+D-1\right)\left(g\left(K^{-1}(t-D)+D\right)^{p}\right. \\
& \quad+D), \quad n \in \mathbf{Z}_{0+}, \quad \forall t(>D) \in \mathbf{R}_{+}
\end{aligned}
$$

then, from Theorem 2.1, there exists the following limit

$$
\begin{aligned}
& 1=\lim _{n \rightarrow \infty} F_{T x_{n+1}, T x_{n}}(t) \geq \lim _{n \rightarrow \infty} F_{x_{0}, T x_{0}}\left(K^{-n}(t-D)+D\right) \\
& =F_{x_{0}, T x_{0}}\left(\lim _{n \rightarrow \infty} K^{-n}(t-D)+D\right)=F_{x_{0}, T x_{0}}(+\infty)=1 \\
& \quad \forall t(>D) \in \mathbf{R}_{+}
\end{aligned}
$$


so that $x= \pm D / 2$ are also best proximity points in the probabilistic sense. In addition, $F_{T^{2 n} x_{0}, T^{2(n+m)} x_{0}}(t)>1-\lambda$ and $F_{T^{2 n} x_{0}, T^{2(n+m)+1} x_{0}}(t+D)>1-\lambda, \forall \lambda \in(0,1) \cap \mathbf{R}$ and $\forall t \in \mathbf{R}_{+}$from Theorem 2.1[(2.10) and (2.15)] and $\left\{T^{2 n} x_{0}\right\}$ and $\left\{T^{2 n+1} x_{0}\right\}$ are Cauchy, $G$-Cauchy and convergent to $\pm x$, respectively. Note that for $D=0$ and $A \cap B=\{0\}$ then $x=0$ is the unique fixed point from Theorem 2.2 since $\left(\mathbf{R}, F, \Delta_{M}\right)$ is a $G$-complete Menger PM-space.

\section{Main results on best proximity points for generalized $p$-cyclic $\alpha-\psi$-type contractions}

We generalize the concept of $p(\geq 2)$-cyclic $\alpha$ - $\psi$-type contractions as follows:

Definition 3.1 Let $(X, F)$ be a PM-space and let $A_{i}$ be nonempty subsets of $X, \forall i \in \bar{p}$ such that $D=d\left(A_{i}, A_{i+1}\right)$ is the distance in-between adjacent subsets, $\forall i \in \bar{p}$. Then, $T$ : $\bigcup_{i \in \bar{p}} A_{i} \rightarrow \bigcup_{i \in \bar{p}} A_{i}$ is a generalized $p(\geq 2)$-cyclic $\alpha$ - $\psi$-type contraction if $T\left(A_{i}\right) \subseteq A_{i+1}, \forall i \in \bar{p}$ and there exist two functions $\alpha:\left(\bigcup_{i \in \bar{p}} A_{i}\right) \times\left(\bigcup_{i \in \bar{p}} A_{i}\right) \times \mathbf{R}_{+} \rightarrow \mathbf{R}_{0+} \quad$ and $\psi \in \Psi$ satisfying the following inequality:

$$
\alpha(x, y, t)\left(\frac{1}{F_{T x, T y}(\varphi(K t+(1-K) D))}-1\right) \leq \psi_{0}(x, y, T x, T y, \varphi(t))
$$

where

$$
\begin{gathered}
\psi_{0}(x, y, T x, T y, \varphi(t))=\max \left(\psi\left(\frac{1}{F_{x, y}(\varphi(t))}-1\right),\right. \\
\psi\left(\frac{1}{F_{x, T x}(\varphi(t))}-1\right), \quad \psi\left(\frac{1}{F_{y, T y}(\varphi(t))}-1\right), \\
\left.\psi\left(\frac{1}{F_{x, T y}(2 \varphi(t))}-1\right), \psi\left(\frac{1}{F_{y, T x}(2 \varphi(t))}-1\right)\right),
\end{gathered}
$$

$\forall(x, y) \in A_{i} \times A_{i+1}, \quad \forall i \in \bar{p}, \quad \forall t(>D) \in \mathbf{R}_{+}$, where $K \in$ $(0,1)$ and $\varphi \in \Phi_{D \hat{D}}$.

An extension of Proposition 2.1 which can be proved under similar arguments follows:

Proposition 3.1 The constraint (3.1), subject to (3.2), is identical to: $\forall(x, y) \in A_{i} \times A_{i+1}, \forall i \in \bar{p}, \forall t(>D) \in \mathbf{R}_{+}$which is also identical, if $\alpha(x, y, t) \neq 0, \quad \forall(x, y) \in A_{i} \times A_{i+1}, \quad \forall i \in \bar{p}$, $\forall t(>D) \in \mathbf{R}_{+}$, to

$$
\begin{gathered}
F_{T x, T y}^{-1}(\varphi(t)) \leq 1+\alpha^{-1}\left(x, y, K^{-1}(t-D)+D\right) \\
\psi_{0}\left(x, y, T x, T y, \varphi\left(K^{-1}(t-D)+D\right)-1\right)
\end{gathered}
$$

Proposition 3.2 Let $(X, F)$ be a PM-space and let $T$ : $\bigcup_{i \in \bar{p}} A_{i} \rightarrow \bigcup_{i \in \bar{p}} A_{i}$ be a generalized p-cyclic $\alpha$ - $\psi$-type contraction with $A_{i} \subset X$ being bounded with $D=$ $d\left(A_{i}, A_{i+1}\right)$ and $\bar{D}=\max _{i \in \bar{p}} \operatorname{diam}\left(A_{i}\right), \forall i \in \bar{p}$ being the distance in-between adjacent subsets, $\forall i \in \bar{p}$. If $\varphi \in \Phi_{D \hat{D}}$, then Proposition 2.2 holds.

Proof It is direct since if $(X, F)$ be a PM-space, $T$ : $\bigcup_{i \in \bar{p}} A_{i} \rightarrow \bigcup_{i \in \bar{p}} A_{i}$ is a generalized $p$-cyclic $\alpha$ - $\psi$-type contraction with $A_{i} \subset X$ being bounded, $D=d\left(A_{i}, A_{i+1}\right)$ and $\bar{D}=\max _{i \in \bar{p}} \operatorname{diam}\left(A_{i}\right)$ being the distance in-between adjacent subsets, $\forall i \in \bar{p}$ and $\varphi \in \Phi_{D \hat{D}}$, then:

$$
\begin{aligned}
& F_{x, y}^{-1}\left(\varphi\left(K^{-1}(t-D)+D\right)\right)=F_{x, y}\left(\varphi\left(K^{-1}(t-D)+D\right)\right)=1 \\
& F_{x, T x}^{-1}\left(\varphi\left(K^{-1}(t-D)+D\right)\right)=F_{x, T x}\left(\varphi\left(K^{-1}(t-D)+D\right)\right)=1 \\
& F_{y, T y}^{-1}\left(\varphi\left(K^{-1}(t-D)+D\right)\right)=F_{y, T y}\left(\varphi\left(K^{-1}(t-D)+D\right)\right)=1 \\
& F_{x, T y}^{-1}\left(2 \varphi\left(K^{-1}(t-D)+D\right)\right)=F_{x, T x}\left(2 \varphi\left(K^{-1}(t-D)+D\right)\right)=1 \\
& F_{y, T x}^{-1}\left(2 \varphi\left(K^{-1}(t-D)+D\right)\right)=F_{y, T y}\left(2 \varphi\left(K^{-1}(t-D)+D\right)\right)=1
\end{aligned}
$$

for $t \in(D+2 \bar{D}, \infty)$ so that it follows in a similar way as in the proof of Proposition 2.2 that

$$
\begin{aligned}
& \psi_{0}\left(x, y, T x, T y, \varphi\left(K^{-1}(t-D)+D\right)\right)=\psi_{0}(x, y, T x, T y, 0) \\
& \quad=0
\end{aligned}
$$

for $t \in(D+2 \bar{D}, \infty)$ since $K \in(0,1)$, the distance distribution function $F: \mathbf{R} \rightarrow[0,1]$ is non-decreasing and left-continuous, $\quad T\left(A_{i}\right) \subseteq T\left(A_{i+1}\right), \quad \forall i \in \bar{p}$, $K^{-1}(t-D)+D>2 \bar{D}+D$, and

$$
2 \varphi\left(K^{-1}(t-D)+D\right)>\varphi\left(K^{-1}(t-D)+D\right)>\varphi(2 \bar{D}+D)
$$

since $\varphi(t)$ is strictly increasing.

$F_{T x, T y}(\varphi(t)) \geq \frac{\alpha\left(x, y, K^{-1}(t-D)+D\right)}{\alpha\left(x, y, K^{-1}(t-D)+D\right)+\psi_{0}\left(x, y, T x, T y, \varphi\left(K^{-1}(t-D)+D\right)-1\right)}$, 
The $\alpha$-admissibility property has the same sense as in the case of $\alpha-\psi$-type contractions and cyclic contractions, that is:

Definitions 3.2 If $(X, F)$ a PM-space then an $\alpha$ - $\psi$-type generalized contraction $T: X \rightarrow X$ (respectively, a generalized $p$-cyclic $\alpha$ - $\psi$-type contraction $T: \bigcup_{i \in \bar{p}} A_{i} \rightarrow \bigcup_{i \in \bar{p}} A_{i}$ ) is $\alpha$-admissible if $\alpha: X \times X \times \mathbf{R}_{+} \rightarrow c l \mathbf{R}_{0+}$ satisfies Definition 2.2 (1) (respectively, Definition 2.2 (2)).

Parallel results to those in Theorem 2.1 and Corollary 2.1 are stated in the following compacted result:

Theorem 3.1 Let $(X, F, \Delta)$ be a G-complete PM-space and let $T: \bigcup_{i \in \bar{p}} A_{i} \rightarrow \bigcup_{i \in \bar{p}} A_{i}$ be a generalized $p$-cyclic $\alpha$ $\psi$-type contraction satisfying the following conditions:

1. $D=d\left(A_{i}, A_{i+1}\right)>0$ and $\operatorname{diam}\left(A_{i}\right)>\hat{D}-D, \forall i \in \bar{p}$,

2. $T$ is $\alpha$-admissible,

3. there exists $x_{0} \in \bigcup_{i \in \bar{p}} A_{i}$ such that $\alpha\left(x_{0}, T x_{0}, t\right) \geq 1$, $\forall t \in \mathbf{R}_{+}$,

4. if $\left\{x_{n}\right\}(\subset X) \rightarrow x$ is a convergent sequence generated by the Picard iteration $x_{n+1}=T x_{n}, \forall n \in \mathbf{Z}_{0+}$ for $a$ given initial condition $x_{0} \in \bigcup_{i \in \bar{p}} A_{i}$, such that $\alpha\left(x_{n}, T x_{n}, t\right) \geq 1, \forall n \in \mathbf{Z}_{0+}, \forall t \in \mathbf{R}_{+}$ then $\alpha\left(x_{n}, x, t\right) \geq 1, \forall n \in \mathbf{Z}_{0+}, \forall t \in \mathbf{R}_{+}$.

Then, the following properties hold:

1. If $\varphi \in \Phi_{D \hat{D}}$ for some $\hat{D}(\geq D) \in \mathbf{R}_{0+}$

2. If $\varphi \in \Phi_{0}$ and $\bigcap_{i \in \bar{p}} A_{i} \neq \emptyset$ then $\left\{T^{n} x_{0}\right\} \subset \bigcup_{i \in \bar{p}} A_{i}$ and $\left\{T^{n p+j} x_{0}\right\} \subset A_{i+j}$ are both Cauchy and G-Cauchy convergent sequences to a limit point $x \in \bigcap_{i \in \bar{p}} c l A_{i}$. If $A_{i}$ are closed for $i \in \bar{p}$ then $x=T x=T^{p} x$, that is, it is a fixed point of the self-mappings $T: \bigcup_{i \in \bar{p}} A_{i} \rightarrow$ $\bigcup_{i \in \bar{p}} A_{i} \quad$ and $\quad T^{p}\left(\equiv T \circ T^{p-1}\right): \bigcup_{i \in \bar{p}} A_{i} \mid A_{k} \rightarrow A_{k}$, $\forall k \in \bar{p}, \forall x_{0} \in A_{i}, \forall i \in \bar{p}, \forall j \in \overline{p-1} \cup\{0\}$.

3. If $A_{i}$ is closed, $\forall i \in \bar{p}$ and $\bigcap_{i \in \bar{p}} A_{i}=\emptyset$ then if $x_{0} \in A_{i}$ for any $i \in \bar{p}$ then there is a limit cycle $\left(\bar{x}_{i}, T \bar{x}_{i}, \ldots, T^{p-1} \bar{x}_{i}\right)$, to which the sequence $\left\{T^{n} x_{0}\right\}$ converges, with $T^{p} \bar{x}_{i}=\bar{x}_{i}, \bar{x}_{i} \in A_{i}, \bar{x}_{i+j}=T^{j} \bar{x}_{i} \in A_{i+j}$, $\forall i \in \bar{p}, \forall j \in \overline{p-1} \cup\{0\}$ being a fixed point of the composite self-mapping $T^{p}: \bigcup_{i \in \bar{p}} A_{i} \mid A_{k} \rightarrow A_{k}, \forall k \in \bar{p}$, and also a quasi-best proximity point of $T: \bigcup_{i \in \bar{p}} A_{i} \rightarrow \bigcup_{i \in \bar{p}} A_{i}$. The subsequence $\left\{T^{n p+j} x_{0}\right\}$ in $A_{i+j}$ converges to $x_{i+j}=T^{j} x_{i}, \forall j \in \bar{p}$ if $x_{0} \in A_{i}$ for any given $i \in \bar{p}$. Furthermore,

$$
\begin{aligned}
& \lim _{n \rightarrow \infty} F_{T^{n p} x_{0}, T^{(n+m) p} x_{0}}(\varepsilon)=F_{x_{0}, T x_{0}}(\infty)=1, \quad F_{T^{n p} x_{0}, T^{(n+m) p} x_{0}}(\varepsilon)>1-\lambda \\
& F_{T^{n p j} x_{0}, T^{n p j+1} x_{0}}(\varphi(D)+\varepsilon)>1-\lambda, \\
& \quad \lim _{n \rightarrow \infty} F_{T^{n p j} x_{0}, T^{n p j+1} x_{0}}(\varphi(D)+\varepsilon)=F_{\bar{x}_{i+j}, \bar{x}_{i+j+1}}=1,
\end{aligned}
$$

for any given $\varepsilon \in \mathbf{R}_{+}, \lambda \in(0,1)$ and some $n_{0}=n_{0}$ $(\varepsilon, \lambda), \forall n\left(\geq n_{0}\right) \in \mathbf{Z}_{0+}$.

The following further properties hold if condition (1) is relaxed to $D=d\left(A_{i}, A_{i+1}\right)>0, \forall i \in \bar{p}$ and Conditions 2-4 still hold:

4. If $\varphi \in \Phi_{D}, \bigcap_{i \in \bar{p}} A_{i} \neq \emptyset$ then $\left\{T^{n} x_{0}\right\} \subset \bigcup_{i \in \bar{p}} A_{i}$ and $\left\{T^{n p+j} x_{0}\right\} \subset A_{i+j}$ are both Cauchy and G-Cauchy convergent sequences to a limit point $x \in \bigcap_{i \in \bar{p}} c l A_{i}$, with $c l A_{i}$ being the closure of $A_{i}$. If $A_{i}$ are closed for $i \in \bar{p}$ then $x=T x=T^{p} x$, that is, it is a fixed point of the self-mappings $\quad T: \bigcup_{i \in \bar{p}} A_{i} \rightarrow \bigcup_{i \in \bar{p}} A_{i} \quad$ and $T^{p}\left(\equiv T \circ T^{p-1}\right): \bigcup_{i \in \bar{p}} A_{i} \mid A_{k} \rightarrow A_{k}, \forall k \in \bar{p}, \forall x_{0} \in A_{i}$, $\forall i \in \bar{p}, \forall j \in \overline{p-1} \cup\{0\}$.

5. If $\varphi \in \Phi_{D}, A_{i}$ is closed $\forall i \in \bar{p}$ and $\bigcap_{i \in \bar{p}} A_{i}=\emptyset$ then, if $x_{0} \in A_{i}$ for any $i \in \bar{p}$, there is a limit cycle $\left(\bar{x}_{i}, T \bar{x}_{i}, \ldots, T^{p-1} \bar{x}_{i}\right)$, to which the sequence $\left\{T^{n} x_{0}\right\}$ converges, with $T^{p} \bar{x}_{i}=\bar{x}_{i}, \bar{x}_{i} \in A_{i}, \bar{x}_{i+j}=T^{j} \bar{x}_{i} \in A_{i+j}$, $\forall i \in \bar{p}, \forall j \in \overline{p-1} \cup\{0\}$ being a fixed point of the composite self-mapping $T^{p}: \bigcup_{i \in \bar{p}} A_{i} \mid A_{k} \rightarrow A_{k}, \forall k \in \bar{p}$, and being also $a$ best proximity point of $T: \bigcup_{i \in \bar{p}} A_{i} \rightarrow \bigcup_{i \in \bar{p}} A_{i}$. The subsequence $\left\{T^{n p+j} x_{0}\right\}$ in $A_{i+j}$ converges to $x_{i+j}=T^{j} x_{i}, \forall j \in \bar{p}$ if $x_{0} \in A_{i}$ for some $i \in \bar{p}$. Furthermore:

$$
\begin{aligned}
& \lim _{n \rightarrow \infty} F_{T^{n p} x_{0}, T^{(n+m) p} x_{0}}(\varepsilon)=F_{x_{0}, T x_{0}}(\infty)=1, \\
& \quad F_{T^{n p} x_{0}, T^{(n+m) p} x_{0}}(\varepsilon)>1-\lambda \\
& F_{T^{n p j} x_{0}, T^{n p j+1} x_{0}}(\varphi(D)+\varepsilon)>1-\lambda, \\
& \quad \lim _{n \rightarrow \infty} F_{T^{n p j} x_{0}, T^{n p j+1} x_{0}}(\varphi(D)+\varepsilon)=F_{\bar{x}_{i+j}, \bar{x}_{i+j+1}}=1
\end{aligned}
$$

for any given $\varepsilon \in \mathbf{R}_{+}, \lambda \in(0,1)$ and some $n_{0}=n_{0}(\varepsilon, \lambda)$, $\forall n\left(\geq n_{0}\right) \in \mathbf{Z}_{0+}$.

Proof Since $T: \bigcup_{i \in \bar{p}} A_{i} \rightarrow \bigcup_{i \in \bar{p}} A_{i}$ is a $p$-cyclic $\alpha$ - $\psi$-type generalized contraction, $x_{0} \in \bigcup_{i \in \bar{p}} A_{i}, \alpha\left(x_{0}, T x_{0}, t\right) \geq 1$, it is proved by complete induction as in Theorem 2.1 that $\alpha\left(x_{n}, T x_{n}, t\right) \geq 1, \forall n \in \mathbf{Z}_{0+}, \forall t \in \mathbf{R}_{+}$since $\varphi \in \Phi_{D \hat{D}}, \varphi$ is strictly increasing in $(D, \infty)$ with $\varphi(D)=\hat{D} \geq D>0$ and then there exists such that $\min \left(F_{x_{0}, T x_{0}}(\varphi(t)), F_{T x_{0}, T^{2} x_{0}}\right.$ $\left.(\varphi(t)), F_{x_{0}, T^{2} x_{0}}(\varphi(t))\right)>0, \forall t(>D) \in \mathbf{R}_{+}$. Since the distance distribution function is non-decreasing and left-continuous and $K^{-1}>1$,

$$
\begin{aligned}
& \min \left(F_{x_{0}, T x_{0}}(\varphi(t)), F_{T x_{0}, T^{2} x_{0}}(\varphi(t)), F_{x_{0}, T^{2} x_{0}}(\varphi(t))\right) \\
& \quad \geq \min \left(F_{x_{0}, T x_{0}}(\varphi(t)), F_{T x_{0}, T^{2} x_{0}}(\varphi(t)), F_{x_{0}, T^{2} x_{0}}(\varphi(t))\right)>0
\end{aligned}
$$


with $t^{\prime}=K^{-1}(t-D)+D, \forall t(>D) \in \mathbf{R}_{+}$and taking inverses in (3.10):

$0 \leq \psi_{0}\left(x_{0}, T x_{0}, T x_{0}, T^{2} x_{0}, \varphi(t)\right)<+\infty, \quad \forall t(>D) \in \mathbf{R}_{+}$

The cases a-c of the proof of Theorem 2.1 are re-addressed via the changes:

$$
\begin{aligned}
& \psi^{n}\left(F_{x_{0}, T x_{0}}^{-1}\left(\varphi\left(K^{-n+1}(t-D)+D\right)\right)-1\right) \\
& \quad \rightarrow \psi_{0}^{n}\left(x_{0}, T x_{0}, T x_{0}, T^{2} x_{0}, \varphi\left(K^{-n+1}(t-D)+D\right)\right)
\end{aligned}
$$

$$
\begin{aligned}
\psi_{0} & \left(T^{n-1} x_{0}, T^{n} x_{0}, T^{n} x_{0}, T^{n+1} x_{0}, \varphi(t)\right) \\
& =\max \left(\psi\left(\frac{1}{F_{T^{n-1} x_{0}, T^{n} x_{0}}(\varphi(t))}-1\right), \psi\left(\frac{1}{F_{T^{n-1} x_{0}, T^{n} x_{0}}(\varphi(t))}-1\right),\right. \\
& \times \psi\left(\frac{1}{F_{T^{n} x_{0}, T^{n+1} x_{0}}(\varphi(t))}-1\right), \psi\left(\frac{1}{F_{T^{n-1} x_{0}, T^{n+1} x_{0}}(2 \varphi(t))}-1\right), \\
& \left.\psi\left(\frac{1}{F_{T^{n} x_{0}, T^{n} x_{0}}(2 \varphi(t))}-1\right)\right) \\
& =\max \left(\psi\left(\frac{1}{F_{T^{n-1} x_{0}, T^{n} x_{0}}(\varphi(t))}-1\right), \psi\left(\frac{1}{F_{T^{n} x_{0}, T^{n+1} x_{0}}(\varphi(t))}-1\right),\right. \\
& \left.\psi\left(\frac{1}{F_{T^{n-1} x_{0}, T^{n+1} x_{0}}(2 \varphi(t))}-1\right)\right),
\end{aligned}
$$

$\forall n \in \mathbf{Z}_{+}, \forall t(>D) \in \mathbf{R}_{+}$, from (3.2), and also

$$
\begin{gathered}
\psi_{0}\left(x_{0}, T x_{0}, T^{2} x_{0}, \varphi(t)\right)=\max \left(\psi\left(\frac{1}{F_{x_{0}, T x_{0}}(\varphi(t))}-1\right),\right. \\
\left.\psi\left(\frac{1}{F_{T x_{0}, T^{2} x_{0}}(\varphi(t))}-1\right), \quad \psi\left(\frac{1}{F_{x_{0}, T^{2} x_{0}}(2 \varphi(t))}-1\right)\right)
\end{gathered}
$$

since

$$
\begin{aligned}
& \psi\left(\frac{1}{F_{T^{n} x_{0}, T^{n} x_{0}}(2 \varphi(t))}-1\right)=\psi\left(\frac{1}{F_{T x_{0}, T x_{0}}(2 \varphi(t))}-1\right) \\
& \quad=\psi(0)=0, \quad \forall t \in \mathbf{R}_{0+}
\end{aligned}
$$

since $F_{x, x}(t)=1, \forall t \in \mathbf{R}_{+}$, since $(X, F)$ is a probabilistic metric space, and $\varphi: \mathbf{R} \rightarrow \mathbf{R}_{0+}$ is nonzero, $\forall t \in \mathbf{R}_{+}$for all $x \in X$ can be removed from the evaluation of the maximum in (3.13). Equations (2.8a)-(2.8b) are changed to:

$$
\begin{aligned}
& F_{T^{n} x_{0}, T^{n+1} x_{0}}^{-1}(\varphi(t)) \leq 1+\psi_{0}\left(T^{n-1} x_{0}, T^{n} x_{0}, T^{n+1} x_{0},\right. \\
& \left.\quad\left(\varphi\left(K^{-1}(t-D)+D\right)\right)-1\right) \\
& \leq 1+\psi_{0}^{n}\left(x_{0}, T x_{0}, T^{2} x_{0}, \varphi\left(K^{-n+1}(t-D)+D\right)\right), \\
& \quad \forall t(>D) \in \mathbf{R}_{+}, \quad \forall n \in \mathbf{Z}_{+}
\end{aligned}
$$

using (3.2) with $x=T^{n-1} x_{0}, \quad y=T x=T^{n} x_{0}, \quad \forall n \in \mathbf{Z}_{+}$, equivalently,

$$
\begin{aligned}
& F_{T^{n} x_{0}, T^{n+1} x_{0}}(\varphi(t)) \\
& \quad \geq \frac{1}{1+\psi_{0}^{n}\left(x_{0}, T x_{0}, T^{2} x_{0}, \varphi\left(K^{-n+1}(t-D)+D\right)\right)}, \\
& \quad \forall t(>D) \in \mathbf{R}_{+}, \quad \forall n \in \mathbf{Z}_{+}
\end{aligned}
$$

Since $K^{-1}>1, \quad \lim \varphi(t)=\infty$ then $\lim \left(K^{-n+1}\right.$ $(t-D)+D)=+\infty$ and $\lim _{n \rightarrow \infty} \varphi\left(K^{-n+1}(t \rightarrow+\infty)+D\right)$ $=+\infty, \quad \forall t(>D) \in \mathbf{R}_{+}, \lim _{n \rightarrow \infty} F_{x_{0}, T x_{0}}^{-1} \quad\left(\varphi\left(K^{-n+1}(t-D)\right.\right.$ $+D))=1, \forall t(>D) \in \mathbf{R}_{+}$. Thus, from (3.15),

$\lim _{n \rightarrow \infty} \psi_{0}^{n}\left(x_{0}, T x_{0}, T^{2} x_{0}, \varphi\left(K^{-n+1}(t-D)+D\right)\right)=0$,

$\forall t(>D) \in \mathbf{R}_{+}$

since

$$
\begin{aligned}
& F_{x_{0}, T x_{0}}\left(\varphi\left(K^{-n+1}(t-D)+D\right)\right) \rightarrow 1, \\
& \quad F_{x_{0}, T^{2} x_{0}}\left(\varphi\left(K^{-n+1}(t-D)+D\right)\right) \rightarrow 1, \\
& \quad F_{T^{2} x_{0}, T x_{0}}\left(\varphi\left(K^{-n+1}(t-D)+D\right)\right) \rightarrow 1
\end{aligned}
$$

$$
\begin{gathered}
\psi^{n}\left(F_{x_{0}, T x_{0}}^{-1}\left(\varphi\left(K^{-n+1}(t-D)+D\right)\right)-1\right) \rightarrow 0, \\
\psi^{n}\left(F_{x_{0}, T^{2} x_{0}}^{-1}\left(\varphi\left(K^{-n+1}(t-D)+D\right)\right)-1\right) \rightarrow 0, \\
\psi^{n}\left(F_{T^{2} x_{0}, T x_{0}}^{-1}\left(\varphi\left(K^{-n+1}(t-D)+D\right)\right)-1\right) \rightarrow 0
\end{gathered}
$$

as $n \rightarrow \infty, \forall t(>D) \in \mathbf{R}_{+}$, since $\psi^{n}\left(t_{n}\right) \rightarrow 0$ if $\left\{t_{n}\right\} \rightarrow 0$. Since $\hat{D}=\varphi\left(D^{+}\right)$, one has from Eqs. (3.16a) and (3.16b) that $\lim _{n \rightarrow \infty} F_{T^{n p j} x_{0}, T^{n p j+1} x_{0}}(\varphi(D)+t)=1, \quad \forall t \in \mathbf{R}_{+} \quad$ with $T^{n p j} x_{0} \in A_{i+j}$ and $T^{n p j+1} x_{0} \in A_{i+j+1}, \forall j \in \bar{p}$ if $x_{0} \in A_{i}$ for any given $i \in \bar{p}$ since $\operatorname{diam}\left(A_{i}\right)>\hat{D}-D, \forall i \in \bar{p}$. Then, for any given real constants $\varepsilon \in \mathbf{R}_{+}$and $\lambda \in(0,1)$, there is some $n_{0}=n_{0}(\varepsilon, \lambda) \in \mathbf{Z}_{0+} \quad$ such that $F_{T^{n p j} x_{0}, T^{n j+1} x_{0}}$ $(\varphi(D)+\varepsilon)>1-\lambda, \forall n \geq n_{0}, \forall j \in \bar{p}$ since the distance distribution function is non-decreasing and left-continuous. Thus, (2.10), for $\forall t(>D) \in \mathbf{R}_{+}, \quad$ (2.11), for $D=\hat{D}$ $=\varphi(0)=0$, and (2.12), for $\ell \in \overline{k-1} \cup\{0\}$, and (2.13) obtained in the proof of Theorem 2.1 also hold, for any $\varepsilon \in \mathbf{R}_{+}$and $\lambda \in(0,1), \forall j \in \bar{p}, \forall n\left(\geq n_{0}\right) \in \mathbf{Z}_{0+}, \forall m \in \mathbf{Z}_{+}$ and some $n_{0}=n_{0}(\varepsilon, \lambda) \in \mathbf{Z}_{0+}$. Then, the $p$ sequences $\left\{T^{n p+i} x_{0}\right\}, \forall i \in \bar{p}$ have a unique limit point in $\bigcap_{i \in \bar{p}} A_{i}$ provided that such a set is nonempty and closed (otherwise, it is allocated in the intersection of the corresponding closures) and, from Assertion 1.1, they are both Cauchy and $G$-Cauchy sequences. The limit point is also proved to 
be a fixed point of $T: \bigcup_{i \in \bar{p}} A_{i} \rightarrow \bigcup_{i \in \bar{p}} A_{i}$ and of $T^{p}: \bigcup_{i \in \bar{p}} A_{i} \mid A_{j} \rightarrow A_{j}, \forall j \in \bar{p}$. Hence, Property (2) has been proved. Property (3) is proved from the still valid formulas (2.14)-(2.18). Properties (4) and (5) follow from their still applicable counterparts of Corollary 2.1 for the cases when $\varphi \in \Phi_{D \hat{D}}, \bigcap_{i \in \bar{p}} A_{i} \neq \emptyset$ and, respectively, $\varphi \in \Phi_{D}, A_{i}$ is closed, $\forall i \in \bar{p}$ and $\bigcap_{i \in \bar{p}} A_{i}=\emptyset$.

Theorem 3.2 Let $(X, F, \Delta)$ a G-complete PM-space and let $T: \bigcup_{i \in \bar{p}} A_{i} \rightarrow \bigcup_{i \in \bar{p}} A_{i}$ be a p-cyclic $\alpha$ - $\psi$-type generalized contraction satisfying the following conditions:

1. $D=d\left(A_{i}, A_{i+1}\right)>0, \quad \forall i \in \bar{p}$,

2. $T$ is $\alpha$-admissible,

3. there exists $x_{0} \in \bigcup_{i \in \bar{p}} A_{i}$ such that $\alpha\left(x_{0}, T x_{0}, t\right) \geq 1$, $\forall t \in \mathbf{R}_{+}$,

4. if $\left\{x_{n}\right\}(\subset X) \rightarrow x$ is a sequence generated as $x_{n+1}=T x_{n}, \quad$ such that $\alpha\left(x_{n}, T x_{n}, t\right) \geq 1, \forall n \in \mathbf{Z}_{0+}, \forall t \in \mathbf{R}_{+}$ then $\alpha\left(x_{n}, x, t\right) \geq 1, \forall n \in \mathbf{Z}_{0+}, \forall t \in \mathbf{R}_{+}$.

5. For all $x, y \in \bigcup_{i \in \bar{p}}$ clA $A_{i}$, there is $z \in \bigcup_{i \in \bar{p}}$ clA $A_{i}$ such that $\min \left(\alpha\left(x, T^{k} z, t\right), \alpha\left(y, T^{k} z, t\right)\right) \geq 1, \forall t(>D) \in \mathbf{R}_{+}$for some $k=k(i, j) \in \overline{p-1} \cup\{0\}$ if $x \in c l A_{i}, y \in$ clA $A_{j}$ for any given $i, j \in \bar{p}$.

Then, there is $\bar{x}_{i} \in c l A_{i}$, such that $\bar{x}_{i} \in A_{i}$ if $A_{i}$ is closed, $\forall i \in \bar{p}$, which is the unique best proximity point of $T$ : $\bigcup_{i \in \bar{p}} A_{i} \rightarrow \bigcup_{i \in \bar{p}} A_{i}$ and the unique fixed point of $T^{p}: \bigcup_{i \in \bar{p}} A_{i} \mid A_{k} \rightarrow A_{k}, \forall k \in \bar{p}$. If, in addition, $\varphi \in \Phi_{0}$ with $D=0$ and $\bigcap_{i \in \bar{p}} A_{i}$ is nonempty and closed then there is a unique fixed point of $T: \bigcup_{i \in \bar{p}} A_{i} \rightarrow \bigcup_{i \in \bar{p}} A_{i}$ and the composite self-mappings $T^{p}: \bigcup_{i \in \bar{p}} A_{i} \mid A_{k} \rightarrow A_{k}, \forall k \in \bar{p}$.

Proof Let $u \in c l A_{i}, v \in c l A_{j}$ be best proximity points for any given $i, j \in \bar{k}$ such that $u=T^{n p} u=T^{p} u$ and $v=T^{n p} v=T^{p} v, \forall n \in \mathbf{Z}_{+}$so that there is $z \in \bigcup_{i \in \bar{p}} c l A_{i}$ such that $\min (\alpha(u, z, t), \alpha(v, z, t)) \geq 1, \forall t(>D) \in \mathbf{R}_{+}$. Thus, note that $T^{p n+k} z \notin c l A_{i} \cup c l A_{j}$ and $T^{k+1} z \notin A_{l}$ if $z \in$ $A_{l}$ for any $\ell \in \bar{p}$ if $D>0$.

Note that $\psi^{n p-1}\left(a_{n p-1}(k)\right) \rightarrow 0$ as $a_{n p-1}(t, k, u, z)=$ $\frac{1}{F_{u, T^{m}}\left(\varphi\left(K^{-(n p+k)}(t-D)+D\right)\right)}-1 \rightarrow 0 \quad$ as $n \rightarrow \infty$ for all $t(>D) \in \mathbf{R}_{+}$which holds since $F_{u, T^{m} z}\left(\varphi\left(K^{-n p}(t-D)\right.\right.$ $+D)) \rightarrow 1$ as $n \rightarrow \infty$ for all $\forall t(>D) \in \mathbf{R}_{+}$since $\left(K^{-n p}(t-D)+D\right) \rightarrow \infty$ for all $t(>D) \in \mathbf{R}_{+}$as $n \rightarrow \infty$ and since $\varphi(t)>D$ from (2.21), from the properties $\varphi(t)=$ 0 for $t<D, \varphi(D) \geq D$, and $\varphi(t)>D$ for $\forall t(>D) \in \mathbf{R}_{+}$ since $\varphi: \mathbf{R}_{0+} \rightarrow \mathbf{R}_{0+}$ is strictly increasing in $[D, \infty)$. As a result, $F_{u, T^{p n+m} z}(t)=F_{v, T^{p n+\ell_{z}}}(t)=1, \forall t(>D) \in \mathbf{R}_{+}$. Let $u, v \in c l A_{i}$ be best proximity points for any given $i \in \bar{k}$ such that $u=T^{p} u$ and $v=T^{p} v$. Then, one gets from (3.19) to (3.20) that, if $\alpha(u, v, t) \geq 1$ for any $t \in \mathbf{R}_{+}$then since and since $T$ is $\alpha$-admissible and $\min \left(\alpha\left(x, T^{k} z, t\right), \alpha\left(y, T^{k}\right.\right.$ $z, t)) \geq 1$

$$
\begin{aligned}
& \frac{1}{F_{T^{n p} u, T^{n p} v}(\varphi(t))}-1=\frac{1}{F_{T^{p} u, T^{p} v}(\varphi(t))}-1=\frac{1}{F_{u, v}(\varphi(t))}-1 \\
& \leq \sup _{n \in Z_{0+}}\left[\alpha^{-1}\left(u, T^{n p+k} z, t\right)\right] \psi_{0}^{n p-1}\left(u, T^{k} z, T u, T^{k+1} v,\right. \\
& \left.\varphi\left(K^{-n p}(t-D)+D\right)\right), \quad \forall t(>D) \in \mathbf{R}_{+} .
\end{aligned}
$$

Note also that

$$
\begin{aligned}
& \limsup _{n \rightarrow \infty} \psi_{0}^{n p-1}\left(u, z, T u, T^{k} z, \varphi\left(K^{-n p}(t-D)+D\right)\right) \\
& =\max \left[\limsup _{n \rightarrow \infty} \psi\left(\frac{1}{F_{u, z}\left(\varphi\left(t_{n}^{\prime}\right)\right)}-1\right)\right. \\
& \limsup _{n \rightarrow \infty} \psi\left(\frac{1}{F_{u, T u}\left(\varphi\left(t_{n}^{\prime}\right)\right)}-1\right), \limsup _{n \rightarrow \infty} \psi\left(\frac{1}{F_{z, T z}\left(\varphi\left(t_{n}^{\prime}\right)\right)}-1\right), \\
& \left.\limsup _{n \rightarrow \infty} \psi\left(\frac{1}{F_{u, T z}\left(2 \varphi\left(t_{n}^{\prime}\right)\right)}-1\right), \limsup _{n \rightarrow \infty} \psi\left(\frac{1}{F_{T z, T u}\left(2 \varphi\left(t_{n}^{\prime}\right)\right)}-1\right)\right] \\
& \leq \max _{n \rightarrow \infty}\left[\limsup _{n \rightarrow \infty}\left(\frac{1}{F_{u, z}\left(\varphi\left(t_{n}^{\prime}\right)\right)}-1\right)\right. \\
& \limsup _{n \rightarrow \infty} \psi\left(\frac{1}{F_{u, T u}\left(\varphi\left(t_{n}^{\prime}\right)\right)}-1\right), \limsup _{n \rightarrow \infty} \psi\left(\frac{1}{F_{z, T z}\left(\varphi\left(t_{n}^{\prime}\right)\right)}-1\right), \\
& \left.\limsup _{n \rightarrow \infty} \psi\left(\frac{1}{F_{u, T z}\left(2 \varphi\left(t_{n}^{\prime}\right)\right)}-1\right), \limsup _{n \rightarrow \infty} \psi\left(\frac{1}{F_{T z, T u}\left(2 \varphi\left(t_{n}^{\prime}\right)\right)}-1\right)\right] \\
& \leq \max \left[\limsup _{n \rightarrow \infty} \psi\left(\frac{1}{F_{u, z}\left(\varphi\left(t_{n}^{\prime}\right)\right)}-1\right) \lim _{n \rightarrow \infty} \sup \psi\left(\frac{1}{F_{u, T u}\left(\varphi\left(t_{n}^{\prime}\right)\right)}-1\right),\right. \\
& \left.\limsup _{n \rightarrow \infty} \psi\left(\frac{1}{F_{z, T z}\left(\varphi\left(t_{n}^{\prime}\right)\right)}-1\right)\right]=\psi(0)=0, \forall t(>D) \in \mathbf{R}_{+}
\end{aligned}
$$

where $t_{n}^{\prime}=t_{n}^{\prime}(t)=K^{-n p}(t-D)+D$ since

$$
\begin{aligned}
& F_{u, T z}\left(2 \varphi\left(t_{n}^{\prime}\right)\right) \geq \Delta_{M}\left(F_{u, z}\left(\varphi\left(t_{n}^{\prime}\right)\right), \quad F_{T z, z}\left(\varphi\left(t_{n}^{\prime}\right)\right)\right) \\
& \quad \geq \min \left(F_{u, z}\left(\varphi\left(t_{n}^{\prime}\right)\right), F_{T z, z}\left(\varphi\left(t_{n}^{\prime}\right)\right)\right)
\end{aligned}
$$

$$
\begin{aligned}
& F_{T u, T z}\left(2 \varphi\left(t_{n}^{\prime}\right)\right) \geq \Delta_{M}\left(F_{u, T u}\left(\varphi\left(t_{n}^{\prime}\right)\right), F_{T z, u}\left(\varphi\left(t_{n}^{\prime}\right)\right)\right) \\
& \quad \geq \min \left(F_{u, T u}\left(\varphi\left(t_{n}^{\prime}\right)\right), F_{T z, u}\left(\varphi\left(t_{n}^{\prime}\right)\right)\right)
\end{aligned}
$$

and, in the same way,

$$
\begin{aligned}
& \lim _{n \rightarrow \infty} \sup _{0}^{n p-1}\left(v, z, T v, T^{k} z, \varphi\left(K^{-n p}(t-D)+D\right)\right)=0 \\
& \forall t(>D) \in \mathbf{R}_{+}
\end{aligned}
$$

so that $t_{n}^{\prime} \rightarrow+\infty$ as $n \rightarrow \infty, \forall t(>D) \in \mathbf{R}_{+}$. Then, it follows from (3.19) and (3.20) that

$F_{u, T^{p+k_{z}}}(t)=F_{v, T^{p n+k_{z}}}(t)=1, \quad \forall t(>D) \in \mathbf{R}_{+}$

$F_{u, T^{p n+k} z}(t)=F_{v, T^{n+k_{z}}}(t)=0, \quad \forall t(\leq D) \in \mathbf{R}_{+}$ 
since $\psi^{n p-1}\left(a_{n p-1}(t, k, u, z)\right) \rightarrow 0$ as $a_{n p-1}(t, k, u, z)=$ $\frac{1}{F_{u, T^{k}}\left(\varphi\left(K^{-(n p+k)}(t-D)+D\right)\right)}-1 \rightarrow 0 \quad$ as $\quad n \rightarrow \infty \quad$ for all $t(>D) \in R_{+}$which holds since $F_{u, T^{k} z}\left(\varphi\left(K^{-n p}(t-D)\right.\right.$ $+D)) \rightarrow 1$ as $n \rightarrow \infty$ for all $\forall t(>D) \in \mathbf{R}_{+}$since $\left(K^{-n p}(t-D)+D\right) \rightarrow \infty$ for all $t(>D) \in \mathbf{R}_{+}$as $n \rightarrow \infty$ and since $\varphi(t)>D$ from (2.21), from the properties $\varphi(t)=$ 0 for $t<D, \varphi(D) \geq D$, and $\varphi(t)>D$ for $t>D$ since $\varphi$ : $\mathbf{R} \rightarrow \mathbf{R}_{0+}$ is strictly increasing in $[D, \infty)$. The same conclusion arises by replacing $u$ by $v$. Thus, $F_{u, v}(\varphi(t))=F_{T^{p} u, T^{p} v}(\varphi(t))=1, \forall t \in \mathbf{R}_{+}$and then $u=v$ is a unique fixed point of $T^{p}: \bigcup_{j \in \bar{p}} A_{j} \mid A_{i} \rightarrow A_{i}$ for any arbitrary given $i \in \bar{p}, u \in c l A_{i}$ and $T u \in c l A_{i+1}$ are unique adjacent best proximity points. If the intersection of the closures of the subsets $A_{i}$ is nonempty then the unique adjacent best proximity points coincide in a unique fixed point which follows in the same way as its counterpart in the proof of Theorem 2.2.

Example 3.1 Example 2.1 is revisited under the constraint (3.1) subject to (3.2) with the same distance distribution function and identical $\alpha(x, y, t)$ with $\varphi(t)=\psi(t)=t$ and making $x \rightarrow x_{0}, \quad y \rightarrow T x_{0}, T y \rightarrow T x_{0}^{2}$ for any arbitrary $x_{0} \in \mathbf{R}$, we get $\left\{x_{2 n}=T^{2 n} x_{0}\right\} \rightarrow x,\left\{x_{2 n+1}=T^{2 n+1} x_{0}\right\} \rightarrow$ $-x$ with $x=D$ if $x_{0} \in A$ and $x=-D$ if $x_{0} \in B$, where

$$
\begin{gathered}
\psi_{0}\left(x_{0}, T x_{0}, T x_{0}, T^{2} x_{0}, t\right)=\max \left(\psi\left(\frac{1}{F_{x_{0}, T x_{0}}(t)}-1\right),\right. \\
\psi\left(\frac{1}{F_{T x_{0}, T^{2} x_{0}}(t)}-1\right), \psi\left(\frac{1}{F_{x_{0}, T^{2} x_{0}}(2 t)}-1\right), \\
\left.\psi\left(\frac{1}{F_{T x_{0}, T x_{0}}(2 t)}-1\right)\right), \quad \forall t(>D) \in R_{+}
\end{gathered}
$$

$$
\begin{aligned}
& \psi_{0}\left(T^{n} x_{0}, T^{n+1} x_{0}, T^{n+1} x_{0}, T^{n+2} x_{0}, t\right) \\
& \quad=\max \left(\psi\left(\frac{1}{F_{T^{n} x_{0}, T^{n+1} x_{0}}(t)}-1\right), \psi\left(\frac{1}{F_{T^{n+1} x_{0}, T^{n+2} x_{0}}(t)}-1\right),\right. \\
& \left.\psi\left(\frac{1}{F_{T^{n} x_{0}, T^{n+2} x_{0}}(2 t)}-1\right), \quad \psi\left(\frac{1}{F_{T^{n+1} x_{0}, T^{n+1} x_{0}}(2 t)}-1\right)\right), \\
& \quad \forall n \in \mathbf{Z}_{0+}, \quad \forall t(>D) \in \mathbf{R}_{+}
\end{aligned}
$$

Since $\left\{T^{n} x_{0}\right\},\left\{T^{n+2} x_{0}\right\} \rightarrow \pm x$ and $\left\{T^{n+1} x_{0}\right\} \rightarrow \mp x$, $\lim _{n \rightarrow \infty} \psi_{0}\left(T^{n} x_{0}, T^{n+1} x_{0}, T^{n+1} x_{0}, T^{n+2} x_{0}, t\right)=0$

$\forall t\left(\in \mathbf{R}_{+}\right)>D$, since

$$
\begin{aligned}
& \lim _{n \rightarrow \infty} F_{T^{n} x_{0}, T^{n+1} x_{0}}(t)=\lim _{n \rightarrow \infty} F_{T^{n+1} x_{0}, T^{n+2} x_{0}}(t) \\
& =\lim _{n \rightarrow \infty} F_{T^{n+1} x_{0}, T^{n+2} x_{0}}\left(t^{\prime}\right)=\lim _{n \rightarrow \infty} F_{T^{n+1} x_{0}, T^{n+1} x_{0}}\left(t^{\prime}\right)=1, \\
& \forall t(>D) \in \mathbf{R}_{+}, \quad \forall t^{\prime} \in \mathbf{R}_{+}
\end{aligned}
$$

Authors' contributions Both authors contributed equally to all the parts of this manuscript.

Acknowledgements The first author is grateful to the Spanish Government for its support with Grant DPI2012-30651, and to the Basque Government for its support through Grant IT378-10.

\section{Compliance with ethical standards}

Competing interests The authors declare that they have no competing interests.

Open Access This article is distributed under the terms of the Creative Commons Attribution 4.0 International License (http://crea tivecommons.org/licenses/by/4.0/), which permits unrestricted use, distribution, and reproduction in any medium, provided you give appropriate credit to the original author(s) and the source, provide a link to the Creative Commons license, and indicate if changes were made.

\section{References}

1. Schweizer, B., Sklar, A.: Probabilistic Metric Spaces. NorthHolland, Amsterdam (1983)

2. Pap, E., Hadzic, O., Mesiar, R.: A fixed point theorem in probabilistic metric spaces and an application. J. Math. Anal. Appl. 202, 433-439 (1996)

3. Sehgal, V.M., Bharucha-Reid, A.T.: Fixed points of contraction mappings on probabilistic metric spaces. Theory Comput. Syst. 6(1), 97-102 (1972)

4. Schweizer, B., Sklar, A.: Statistical metric spaces. Pac. J. Math. 10, 313-334 (1960)

5. Eldred, A.A., Veeramani, P.: Existence and convergence of best proximity points. J. Math. Anal. Appl. 323, 1001-1006 (2006)

6. De la Sen, M.: Linking contractive self-mappings and cyclic Meir-Keeler contractions with Kannan self-mappings. Fixed Point Theory Appl. 1, 572057 (2010). doi:10.1155/2010/572057. (Article ID: 572057)

7. Choudhury, B.S., Das, K., Bhandari, S.K.: Fixed point theorem for mappings with cyclic contraction in Menger spaces. Int. J. Pure Appl. Sci. Technol. 4(1), 1-9 (2011)

8. Choudhury, B.S., Das, K., Bhandari, S.K.: Cyclic contraction result in 2- Menger space. Bull. Int. Math. Virtual Inst. 2(1), 223-234 (2012)

9. Beg, I., Abbas, M.: "Fixed point and best approximation in Menger convex metric spaces", Archivum Mathematicum (BRNO). Tomus 41, 389-397 (2005)

10. Beg, I., Latif, A., Abbas, M.: Coupled fixed points of mixed monotone operators on probabilistic Banach spaces. Arch. Math. 37, 1-8 (2001)

11. Mihet, D.: Altering distances in probabilistic Menger spaces. Nonlinear Anal. 71, 2734-2738 (2009)

12. Mihet, D.: A Banach contraction theorem for fuzzy metric spaces. Fuzzy Sets Syst. 144, 431-439 (2004)

13. Sedghi, S., Choudhury, B.S., Shobe, N.: Strong common coupled fixed point result in fuzzy metric spaces. J. Phys. Sci. 17, 1-9 (2013)

14. Wairojjana, N., Dosenovic, T., Rakic, D., Gopal, D., Kumam, P.: An altering distance function in fuzzy metric fixed point theorems. Fixed Point Theory Appl. 2015, 69 (2015)

15. Choudhury, B.S., Das, K.P.: A new contraction principle in Menger spaces. Acta Math.Sin. (Engl. Ser.) 24(8), 1379-1386 (2008) 
16. Gopal, D., Abbas, M., Vetro, C.: Some new fixed point theorems in Menger PM-spaces with application to Volterra type integral equation. Appl. Math. Comput. 232, 955-967 (2014)

17. De la Sen, M., Agarwal, R.P., Nistal, N.: "Non-expansive and potentially expansive properties of two modified p-cyclic selfmaps in metric spaces. J. Nonlinear Convex Anal. 14(4), 661-686 (2013)

18. De la Sen, M., Agarwal, R.P.: Fixed point-type results for a class of extended cyclic self-mappings under three general weak contractive conditions of rational type. Fixed Point Theory Appl. 2011, 102 (2011). doi:10.1186/1687-1812-2011-102

19. De la Sen, M.: On best proximity point theorems and fixed point theorems for $p$-cyclic hybrid self-mappings in Banach spaces. Abstr. Appl. Anal. (2013). doi:10.1155/2013/1831747. (Article ID: 183174)

20. Karpagam, S., Agrawal, S.: Best proximity point theorems for p-cyclic Meir-Keeler contractions. Fixed Point Theory Appl. (2009) (Article ID: 197308)

21. Suzuki, T.: Some notes on Meir-Keeler contractions and Lfunctions. Bull. Kyushu Inst. Technol. 53, 12-13 (2006)

22. Di Bari, C., Suzuki, T., Vetro, C.: Best proximity points for cyclic Meir-Keeler contractions. Nonlinear Anal. Theory Methods Appl. 69(11), 2790-3794 (2008)

23. Rezapour, S., Derafshpour, M., Shahzad, N.: On the existence of best proximity points of cyclic contractions. Adv. Dyn. Syst. Appl. 6(1), 33-40 (2011)

24. Derafshpour, M., Rezapour, S., Shahzad, N.: Best proximity points of cyclic $\varphi$-contractions on reflexive Banach spaces. Fixed Point Theory Appl. 2010, 33-40 (2010). (Article ID: 946178)

25. Al-Thagafi, M.A., Shahzad, N.: Convergence and existence results for best proximity points. Nonlinear Anal. Theory Methods Appl. 70(10), 3665-3671 (2009)

26. De la Sen, M.: Stable iteration procedures in metric spaces which generalize a Picard-type iteration. Fixed Point Theory Appl. (2010). doi:10.1155/2010/953091. (Article ID: 953091)
27. Karpagam, S., Agrawal, S.: Best proximity point theorems for p-cyclic Meir-Keeler contractions. Fixed Point Theory Appl. (2009) (Article ID 197308)

28. Dutta, P.N., Choudhury, B.S., Das, K.: Some fixed point results in Menger spaces using a control function. Surv. Math. Appl. 4, 41-52 (2009)

29. Chang, S.S., Cho, Y.J., Kang, S.M.: Nonlinear Operator Theory in Probabilistic Metric Spaces. Nova Science Publishers, New York (2001)

30. De la Sen, M., Karapınar, E.: Some results on best proximity points of cyclic contractions in probabilistic metric spaces. J. Funct. Spaces 2015, 11 (2015). (Article ID: 470574)

31. Chen, C.M., Chang, T.H., Juang, K.S.: Common fixed point theorems for the stronger Meir-Keeler cone-type function in cone-ball metric spaces. Appl. Math. Lett. 25(4), 692-697 (2012)

32. Chen, C.M.: Common fixed point theorems in complete generalized metric spaces. J. Appl. Math. 2012, 14 (2012). (Article ID: 945915)

33. Alotaibi, A., Mursaleen, M., Sharma, S.K., Mohiuddine, S.A.: Sequence spaces of fuzzy numbers defined by a Musielak-Orlicz function. Filomat 29(7), 1461-1468 (2015)

34. Wang, S.: On $\varphi$-contractions in partially ordered fuzzy metric spaces. Fixed Point Theory Appl. 2015, 233 (2015)

35. Batool, A., Kamran, T., Jang, S.Y., Park, C.: Generalized $\varphi$-weak contractive fuzzy mappings and related fixed point results on complete metric space. J. Comput. Anal. Appl. 21(4), 729-737 (2016)

36. Shen, Y., Wang, F.: A fixed point approach to the Ulam stability of fuzzy differential equations under generalized differentiability. J. Intell. Fuzzy Syst. 30(6), 3253-3260 (2016)

37. Alotaibi, A., Mursaleen, M., Mohiuddine, S.A.: Some fixed point theorems for Meir-Keeler condensing operators with applications to integral equations. Bull. Belg. Math. Soc. Simon Stevin 22(4), 529-541 (2015) 